\title{
Verification of iterative matrix solutions for multipoint kinetics equations
}

Giuseppe Palmiotti, Jean Tommasi

October 2018

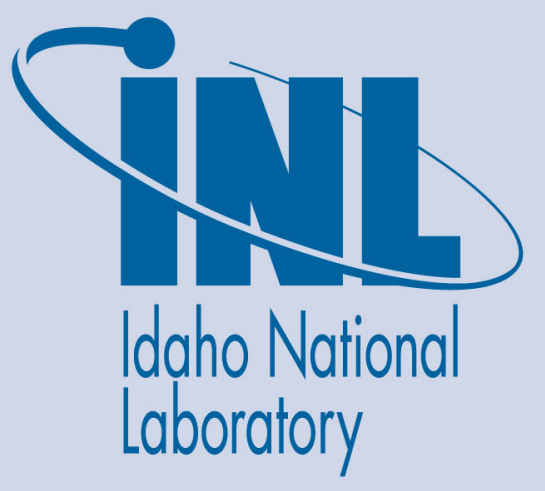

The INL is a U.S. Department of Energy National Laboratory operated by Battelle Energy Alliance 


\title{
Verification of iterative matrix solutions for multipoint kinetics equations
}

\author{
Giuseppe Palmiotti, Jean Tommasi
}

October 2018

Idaho National Laboratory Idaho Falls, Idaho 83415

http://www.inl.gov

Prepared for the

U.S. Department of Energy

Under DOE Idaho Operations Office

Contract Unknown 


\title{
Verification of iterative matrix solutions for multipoint kinetics equations
}

\author{
Jean Tommasi \\ CEA, DEN, DER, SPRC, Cadarache, F-13108 Saint Paul lez Durance, France \\ Giuseppe Palmiotti \\ Idaho National Laboratory, 2525 Fremont Ave. P.O. Box 1625, Idaho Falls, ID 83415-3860 (USA)
}

\begin{abstract}
Multipoint kinetic equation systems have been solved numerically using matrix algebra software and $1^{\text {st }}$ to $4^{\text {th }}$ order implicit schemes based on single-step matrix propagators. These matrix schemes have been validated successfully on demanding point kinetics benchmarks with various prescribed reactivity insertions. Verification tests have also been performed on a simple 3-region fast reactor core model with asymmetric step or ramp reactivity insertions and cross-checked with the multipoint kinetics SACRE code developed at INL and based on existing and validated stiff ODE solver packages. Accurate results can be obtained at a limited time expense. The reason for intriguing results obtained in some transients has been elucidated and linked to the multipoint matrix coefficient interpolation method during the transient.
\end{abstract}

Keywords: multipoint kinetics equations, implicit matrix methods

\section{Introduction}

Multipoint kinetics (MPK) is a description tool for the change in time of the neutron field in a nuclear reactor, intermediate between the point kinetics (PK) description that neglects any change in the spatial distribution of the flux and a full space (3D) + time kinetic description of the reactor. The system is divided in several fissile regions, each considered as a separate point reactor and connected to others by coupling coefficients and neutron generation times. Prompt and delayed neutrons are accounted for. It is believed that, provided the number and extension of the regions have been chosen with physical insight in connection to the response sought for, MPK can capture many features of real kinetic transients.

The first multipoint model was derived by Avery (Avery, 1958) in view of application to the design of a coupled fast-thermal reactor. Spatial integrations on regions, to obtain the multipoint variables and coefficients, are performed after multiplication by weight functions with physical meaning. Avery used weight functions connected to the classical adjoint flux (neutron importance). An alternate form was derived by Kobayashi (Kobayashi, 1991), using Green functions related to the production of next generation neutrons. Avery's and Kobayashi's models are multipoint in space only, but models multipoint in space and in energy have been proposed also (Bosio et al., 2001; Ravetto et al, 2004). Avery and Kobayashi provided a technique to compute the coupling coefficients between zones using deterministic codes, but another possibility is to use pragmatic definitions for the coupling coefficients, based on probabilities, opening the way to their computation by Monte-Carlo estimators (Aufiero et al. 2016; Laureau et al. 2017a\&b). 
We will restrict here to space multipoint models. The goal of the present study was to build a simple and quick, but nevertheless accurate, tool to compute prescribed transients on multipoint core models, with possible further extensions to transients with feedback. Such a tool could be used to gain insight on the quantitative time behaviour of spatially decoupled cores (zero power experimental reactors with very different and coupled regions, large industrial cores). Using matrix algebra software, this tool will be tagged as MATMPK in the following for quick reference. Another incentive to develop MATMPK was to provide comparison terms with the existing MPK code, named SACRE, developed independently at INL (Palmiotti et al., 2018) and help in understanding puzzling results obtained by the latter (Palmiotti et al., 2018, and see below).

The kinetic equations describing the evolution of the neutron flux $\Psi(\vec{r}, E, \vec{\Omega}, t)$ and the concentrations of the $D \geq 1$ families of delayed neutron precursors $C^{(i)}(\vec{r}, t)$ may be written as the following system of $(1+D)$ equations:

$$
\left\{\begin{array}{l}
\frac{1}{v} \frac{\partial \Psi}{\partial t}=\left(\mathbf{F}_{p}-\mathbf{A}\right) \Psi+\sum_{i=1}^{D} \chi_{d}^{(i)} \lambda^{(i)} C^{(i)} \\
\frac{d C^{(i)}}{d t}=\int v_{d}^{(i)} \Sigma_{f} \Psi d E d^{2} \Omega-\lambda^{(i)} C^{(i)}
\end{array}\right.
$$

$\mathbf{F}$ is the operator governing the neutron production by fission, i.e. the sum of $\mathbf{F}_{p}$ (prompt fission production operator) and $\mathbf{F}_{d}$ (delayed fission production operator). $\mathbf{A}$ is the operator grouping the scattering, streaming and collision terms:

$$
\begin{gathered}
\mathbf{F}_{p} \Psi=\chi_{p}(E, \vec{\Omega}) \int d E^{\prime} d^{2} \Omega^{\prime} v_{p}\left(\vec{r}, E^{\prime}, t\right) \Sigma_{f}\left(\vec{r}, E^{\prime}, t\right) \Psi\left(\vec{r}, E^{\prime}, \vec{\Omega}^{\prime}, t\right) \\
\mathbf{F}_{d} \Psi=\sum_{i=1}^{D} \chi_{d}^{(i)}(E, \vec{\Omega}) \int d E^{\prime} d^{2} \Omega^{\prime} v_{d}^{(i)}\left(\vec{r}, E^{\prime}, t\right) \Sigma_{f}\left(\vec{r}, E^{\prime}, t\right) \Psi\left(\vec{r}, E^{\prime}, \vec{\Omega}^{\prime}, t\right) \\
\mathbf{A} \Psi=\vec{\Omega} \cdot \vec{\nabla} \Psi+\Sigma_{t} \Psi-\int d E^{\prime} d^{2} \Omega^{\prime} \Sigma_{s}\left(\vec{r}, E^{\prime} \rightarrow E, \overrightarrow{\Omega^{\prime}} \rightarrow \vec{\Omega}\right) \Psi\left(\vec{r}, E^{\prime}, \vec{\Omega}^{\prime}\right)
\end{gathered}
$$

Fission spectrum normalization is: $\int d E d^{2} \Omega \chi(E, \vec{\Omega})=1$

Eq.(1) is a system of coupled equations for the neutron flux and the precursor concentrations. But hereafter, for quick reference, any equation containing the time derivative of the flux will be called a flux equation, and any equation containing the time derivative of a precursor concentration will be called a precursor equation. In equation systems such as Eq.(1), only one line for the flux equations and one line for the precursor equations will be written; indices will avoid confusion (e.g. the second line in Eq.(1) being indexed by $(i)$, represents $D$ precursor equations)

The classical derivation of PK equations, dating back to (Henry, 1958) is reminded in Appendix. The multipoint equations are derived in Section 2 for Avery's and Kobayashi's models, together with the definition of all the coupling coefficients and the way these coefficients can be approximated for their calculation in deterministic codes. Assuming the coupling coefficients, produced by any suitable method, are available, the PK and MPK variables obey systems of linear ordinary differential equations: $(1+D)$ equations for PK 
and, if $N$ is the number of fissile regions $N(1+D)$ equations for Kobayashi's MPK formulation and $N(N+D)$ equations for Avery's MPK formulation. These linear systems can be cast in a vector-matrix format, and Section 3 presents the derivation of approximate single-step matrix propagators over a time interval $\Delta t$ : those yielding the well-known first (implicit Euler) and second (Crank-Nicolson) order methods, and two more complex ones, based on Newton-Cotes quadrature formulas, resulting in third and fourth order methods. In this same section, a short reminder of the structure and capabilities of the SACRE code developed at INL is provided.

The free and open source software Scilab (Scilab Enterprises, 2012) has been used to code these matrix propagators in order to test and validate them. Scilab is used as a toolbox for fast (compiled) and accurate solvers for matrix algebra operations, here mainly matrix product, inversion and exponentiation. Its interpreted user's language has been used to write scripts performing all ancillary tasks (fill in the matrices, organize the loops and tests, call the built-in pre-compiled functions, print the results).

Section 4 presents the verification of MATMPK on demanding PK benchmarks (Ganapol, 2013), with benchmark 10-digit objective values for three kinds of prescribed reactivity injection (step, ramp, sinusoidal). Attention is given on accuracy vs. time step size, and to CPU times. In Section 5 a simple 3-region fast reactor model with $2 \pi / 3$ rotation symmetry in its reference configuration is defined; the kinetic transients are based on extraction of a single control rod, breaking the symmetry of the core. The coupling coefficients necessary to MATMPK are computed using the ERANOS code system (Ruggieri et al., 2006), and two simple verification tests are performed: a step insertion (the control rod is extracted instantaneously) and a ramp insertion (progressive extraction of the control rod). The step insertion can be validated against an analytical solution obtained by matrix exponentiation; for the ramp insertion, a qualitative match with physical insight is sought for. With respect to the previous PK benchmarks, additional attention is given to shape variations, i.e. the progressive change in the balance of fission rates in the three fissile regions of the core. Finally, Section 6 is devoted to the comparison of results provided by MATMPK and SACRE on a same 3-region problem. In addition, the reason for intriguing results previously obtained by SACRE when modelling the same transient by various methods (PK, MPK with various subdivisions of the reactor into regions) has been elucidated and linked to the multipoint matrix coefficient interpolation method used during the transient.

\section{Derivation of the multipoint equations}

The usual technique (Henry, 1958; Avery, 1958; Komata, 1969; Kobayashi, 1992) is to multiply the kinetics equations Eq.(1) by one or several weight functions and to integrate over space, energy and angle. We shall use the following notation for the functional scalar product so defined:

$$
<f, g>=\int d^{3} r d E d^{2} \Omega f(\vec{r}, E, \vec{\Omega}) g(\vec{r}, E, \vec{\Omega})
$$

The usual derivation of PK equations (Henry, 1958) is recalled in Appendix. The weight function used in this case is a critical adjoint flux, and the lumped parameter obtained from the neutron flux is called the neutron population or amplitude. In the MPK models 
of Kobayashi and Avery, the lumped parameters obtained from the neutron flux are more directly related to region-wise fission source rates.

\subsection{Kobayashi's MPK equations}

The system is partitioned into $N$ distinct fissile regions. $\delta_{n}$ is the function equal to 1 in region $n, 0$ elsewhere. The precursor concentrations, divided into $D$ time families, are split into:

$$
C^{(i)}=\sum_{n=1}^{N} C_{n}^{(i)} \quad \text { with } \quad C_{n}^{(i)}=\delta_{n} C^{(i)} \quad(i=1, \cdots, D)
$$

Each of the $D$ precursor equations is split into $N$ equations (by multiplication by the $\delta_{n}$ ), so that Eq.(1) can be written as the following system of 1 flux equation and $N D$ precursor equations :

$$
\left\{\begin{array}{c}
\frac{1}{v} \frac{\partial \Psi}{\partial t}=\mathbf{F}_{p} \Psi-\boldsymbol{A} \Psi+\sum_{i=1}^{D} \lambda^{(i)} \chi_{d}^{(i)} \sum_{n=1}^{N} C_{n}^{(i)} \\
\frac{d C_{n}^{(i)}}{d t}=\int v_{d}^{(i)} \Sigma_{f n} \Psi d E d^{2} \Omega-\lambda^{(i)} C_{n}^{(i)}
\end{array}\right.
$$

where $\Sigma_{f n}=\delta_{n} \Sigma_{f}$ is the restriction of the fission cross-section to region $n$. For the flux equations a weight function $W_{m}$ is defined for each region and we obtain the following set of $N(1+D)$ equations ( $N$ flux equations and $N D$ precursor equations):

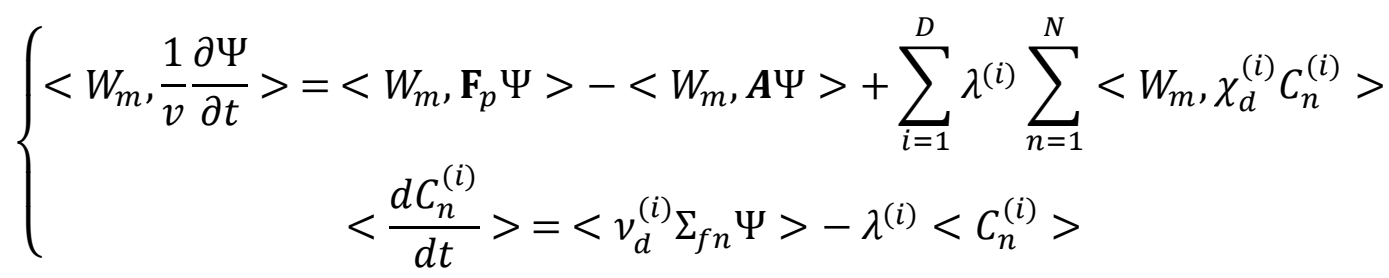

(for $C_{n}^{(i)}$ integration is on space only). The weight function $W_{m}$ chosen by Kobayashi is the function $G_{m}^{+}$obeying the source equation:

$$
\mathbf{A}^{+} G_{m}^{+}=v \Sigma_{f m}
$$

This choice is meant to make the term $\left\langle W_{m}, A \Psi\right\rangle$ in Eq.(9) equal to the fission source in region $m, S_{m}=\left\langle\mathbf{F}_{m} \Psi\right\rangle\left(\mathbf{F}_{m}=\delta_{m} \mathbf{F}\right.$ is the restriction of the production operator to region $m$ ), which will be one of the unknowns of the final MPK set of equations:

$$
<G_{m}^{+}, \mathbf{A} \Psi>=<\mathbf{A}^{+} G_{m}^{+}, \Psi>=<v \Sigma_{f m} \Psi>=<\mathbf{F}_{m} \Psi>=S_{m}
$$

By its definition in Eq.(10), $G_{m}^{+}(\vec{r}, E, \vec{\Omega})$, defined over the whole system, represents the expected value for the number of neutrons produced at next generation in region $m$ for 1 current generation neutron placed at $(\vec{r}, E, \vec{\Omega})$.

The other terms in Eq.(9) can be worked out as follows (definitions for the integral kinetics parameters are given directly in the equations) : 


$$
\begin{gathered}
<G_{m}^{+}, \mathbf{F}_{p} \Psi>=\sum_{n=1}^{N} \frac{<G_{m}^{+},\left(\mathbf{F}_{n}-\mathbf{F}_{d n}\right) \Psi>}{<G_{m}^{+}, \mathbf{F}_{n} \Psi>} \cdot \frac{\left\langle G_{m}^{+}, \mathbf{F}_{n} \Psi>\right.}{\left\langle\mathbf{F}_{n} \Psi>\right.} \cdot\left\langle\mathbf{F}_{n} \Psi>\right. \\
\stackrel{\text { def }}{=} \sum_{n=1}^{N}\left(1-\beta_{m n}\right) k_{m n} S_{n}
\end{gathered}
$$

$k_{m n}$ is the expected number of neutrons produced at next generation in region $m$ for 1 neutron produced at current generation in region $n$ and is called a coupling coefficient; the matrix $\mathrm{K}$ such as $K_{m n}=k_{m n}$ being called the coupling matrix. $\beta_{m n}$ is the fraction of delayed neutrons produced at next generation in region $m$ by neutrons produced at current generation in region $n$ due to delayed neutrons produced at current generation in region $n$.

$$
<G_{m}^{+}, \frac{1}{v} \frac{\partial \Psi}{\partial t}>=\frac{<G_{m}^{+}, \frac{1}{v} \frac{\partial \Psi}{\partial t}>}{<\frac{\partial\left(\mathbf{F}_{m} \Psi\right)}{\partial t}>}<\frac{\partial\left(\mathbf{F}_{m} \Psi\right)}{\partial t}>\stackrel{\text { def }}{=} \ell_{m} \frac{d S_{m}}{d t}
$$

$\ell_{m}$ has the dimensionality of time. It is the ratio of the number of neutrons produced at next generation in region $m$ by excess neutrons originating from the whole system to the increase of production rate in region $m$, and may be considered an average time needed by prompt neutrons to reach region $m$ and generate next generation neutrons.

$$
\begin{gathered}
\sum_{i=1}^{D} \lambda^{(i)} \sum_{n=1}^{N}<G_{m}^{+}, \chi_{d}^{(i)} C_{n}^{(i)}>=\sum_{i=1}^{D} \lambda^{(i)} \sum_{n=1}^{N} \frac{<G_{m}^{+}, \chi_{d}^{(i)} C_{n}^{(i)}>}{<C_{n}^{(i)}>}<C_{n}^{(i)}> \\
\stackrel{\text { def }}{=} \sum_{i=1}^{D} \lambda^{(i)} \sum_{n=1}^{N} k_{m n}^{(i)}<C_{n}^{(i)}>
\end{gathered}
$$

$k_{m n}^{(i)}$ is the expected number of neutrons produced at next generation in region $m$ for 1 delayed neutron of family $i$ produced at current generation in region $n$; we can also group them into matrices $\mathbf{K}^{(i)}$ called delayed coupling matrices.

$$
\left\langle v_{d}^{(i)} \Sigma_{f m} \Psi>=\frac{<v_{d}^{(i)} \Sigma_{f m} \Psi>}{\left\langle\mathbf{F}_{m} \Psi>\right.} \cdot\left\langle\mathbf{F}_{m} \Psi>\stackrel{\text { def }}{=} \beta_{m}^{(i)} S_{m}\right.\right.
$$

$\beta_{m}^{(i)}$ is the raw delayed neutron fraction for family $i$ in region $m$. The $N(1+D)$ Kobayashi multipoint equations are then, keeping the notation $C_{m}^{(i)}$ for $\left\langle C_{m}^{(i)}>\right.$ :

$$
\left\{\begin{array}{c}
\ell_{m} \frac{d S_{m}}{d t}=\left(1-\beta_{m}\right) \sum_{n=1}^{N} k_{m n} S_{n}-S_{m}+\sum_{i=1}^{D} \lambda^{(i)} \sum_{n=1}^{N} k_{m n}^{(i)} C_{n}^{(i)} \\
\frac{d C_{m}^{(i)}}{d t}=\beta_{m}^{(i)} S_{m}-\lambda^{(i)} C_{m}^{(i)}
\end{array}\right.
$$

So far, all the involved coefficients are written using the unknown dynamic flux $\Psi(t)$, but they can be approximated by using instead of $\Psi$ the static flux $\Phi$ of the associated critical problem, solution of

$$
\left(\frac{\mathbf{F}}{k_{c}}-\mathbf{A}\right) \Phi=0
$$


the operators being taken at current time. For the generation times, we assume an asymptotic exponential regime where the derivatives of funtions are proportional to the functions themselves, with identification of the time eigenfunction with the multiplication factor eigenfunction of Eq.(17). This way we obtain the following set of approximate point kinetic parameters for the Kobayashi equations:

$$
\begin{array}{cr}
k_{m n} \approx \frac{\left\langle G_{m}^{+}, \mathbf{F}_{n} \Phi>\right.}{\left\langle\mathbf{F}_{n} \Phi>\right.} & k_{m n}^{(i)} \approx \frac{\left\langle G_{m}^{+}, \mathbf{F}_{d n}^{(i)} \Phi>\right.}{<\mathbf{F}_{d n}^{(i)} \Phi>} \\
\beta_{m n} \approx \frac{\left\langle G_{m}^{+}, \mathbf{F}_{d n} \Phi>\right.}{\left\langle G_{m}^{+}, \mathbf{F}_{n} \Phi>\right.} & \beta_{m}^{(i)} \approx \frac{\left\langle v_{d}^{(i)} \Sigma_{f m} \Phi>\right.}{<\mathbf{F}_{m} \Phi>} \\
\ell_{m} \approx \frac{\left\langle G_{m}^{+}, \frac{1}{v} \Phi>\right.}{\left\langle\mathbf{F}_{m} \Phi>\right.} &
\end{array}
$$

\subsection{Avery's MPK equations}

With respect to the Kobayashi approach, the neutron flux $\Psi$ is also split into a sum of partial fluxes:

$$
\Psi=\sum_{n=1}^{N} \Psi_{n}
$$

Here $\Psi_{n}$ is defined as the partial flux due to only the neutrons (prompt and delayed) produced in region $n$. Hence, the kinetic equations, Eq.(1), become the following system of $N(1+D)$ equations ( $N$ flux equations $+N D$ precursor equations):

$$
\left\{\begin{array}{c}
\frac{1}{v} \frac{\partial \Psi_{n}}{\partial t}=\mathbf{F}_{p n} \Psi-A \Psi_{n}+\sum_{i=1}^{D} \chi_{d}^{(i)} \lambda^{(i)} C_{n}^{(i)} \\
\frac{d C_{n}^{(i)}}{d t}=\int v_{d}^{(i)} \Sigma_{f n} \Psi d E d^{2} \Omega-\lambda^{(i)} C_{n}^{(i)}
\end{array}\right.
$$

In the flux equations, the prompt and delayed fission neutron sources are strictly localized to region $n$, but as operator $\mathrm{A}$ includes spatial derivatives, the $\Psi_{n}$ are non-zero over the whole system (although they may decrease sharply outside region $n$ ). When starting from an initial static critical configuration $\Psi_{0}=\Phi_{0}$, the initial values of the partial fluxes, $\Psi_{n 0}$, are solution of the $n$ sub-critical source problems

$$
\left(\mathbf{A}_{0}-\mathbf{F}_{p n 0}\right) \Psi_{n 0}=\sum_{i=1}^{D} \chi_{d}^{(i)} \lambda^{(i)} C_{n 0}^{(i)} \quad \text { with } \quad C_{n 0}^{(i)}=\frac{v_{d}^{(i)} \Sigma_{f n 0} \Phi_{0}}{\lambda^{(i)}}
$$

Here again, we associate to each region $m=1, \cdots, N$ a weight function $W_{m}$ defined over the whole system and we integrate to obtain the following system of $N(N+D)$ equations $\left(N^{2}\right.$ flux equations $+N D$ precursor equations:

$$
\left\{\begin{aligned}
<W_{m}, \frac{1}{v} \frac{\partial \Psi_{n}}{\partial t}>= & <W_{m}, \mathbf{F}_{p n} \Psi>-<W_{m}, A \Psi_{n}>+\sum_{i=1}^{D} \lambda^{(i)}<W_{m}, \chi_{d}^{(i)} C_{n}^{(i)}> \\
& <\frac{d C_{m}^{(i)}}{d t}>=<v_{d}^{(i)} \Sigma_{f m} \Psi>-\lambda^{(i)}<C_{m}^{(i)}>
\end{aligned}\right.
$$


We could use also here the weight functions defined by Kobayashi in Eq.(10), but Avery followed another path. His choice for the weight function $W_{m}$ is a multiple of the "partial static adjoint flux" $\Phi_{m}^{+}$solution of the source equation

$$
\mathbf{A}^{+} \Phi_{m}^{+}=\frac{\delta_{m}}{k_{c}} \mathbf{F}^{+} \Phi^{+}=\frac{1}{k_{c}} \mathbf{F}_{m}^{+} \Phi^{+}
$$

Where $\Phi^{+}$is a fundamental solution of the associated adjoint critical problem:

$$
\left(\frac{\mathbf{F}^{+}}{k_{c}}-\mathbf{A}^{+}\right) \Phi^{+}=0
$$

(this is the equation adjoint to Eq.(17)). By construction, we have:

$$
\sum_{m=1}^{N} \Phi_{m}^{+}=\Phi^{+}
$$

Then, through simple algebraic manipulations:

$$
<\Phi_{m}^{+}, \mathbf{A} \Psi_{n}>=<\mathbf{A}^{+} \Phi_{m}^{+}, \Psi_{n}>=\frac{1}{k_{c}}<\mathbf{F}_{m}^{+} \Phi^{+}, \Psi_{n}>=\frac{1}{k_{c}}<\Phi^{+}, \mathbf{F}_{m} \Psi_{n}>
$$

The multiplicative coefficient $\alpha_{m}$ in $W_{m}=\alpha_{m} \Phi_{m}^{+}$is chosen to ensure a summation to the fission source of region $m$ as follows:

$$
\begin{gathered}
\frac{\alpha_{m}}{k_{c}} \sum_{n=1}^{N}<\Phi^{+}, \mathbf{F}_{m} \Psi_{n}>=\frac{\alpha_{m}}{k_{c}}<\Phi^{+}, \mathbf{F}_{m} \Psi>=<\mathbf{F}_{m} \Psi> \\
\Rightarrow \quad \alpha_{m}=k_{c} \frac{<\mathbf{F}_{m} \Psi>}{<\Phi^{+}, \mathbf{F}_{m} \Psi>}
\end{gathered}
$$

Then we can define the partial fission sources, which will be part of the unknowns in the final MPK equations, as:

$$
\begin{gathered}
S_{m n}=\frac{\left\langle\mathbf{F}_{m} \Psi>\cdot<\Phi^{+}, \mathbf{F}_{m} \Psi_{n}\right\rangle}{<\Phi^{+}, \mathbf{F}_{m} \Psi>} \\
S_{m}=\sum_{n=1}^{N} S_{m n}=<\mathbf{F}_{m} \Psi>
\end{gathered}
$$

$S_{m n}$ can be interpreted as the part of the fission rate $S_{m}$ due to only the neutrons originated by neutrons produced in region $n$. The first term in the right hand side of the flux equations in Eq.(22) can be developed as:

$$
\begin{gathered}
k_{c}<\Phi_{m}^{+}, \mathbf{F}_{p n} \Psi>\frac{\left\langle\mathbf{F}_{m} \Psi>\right.}{<\Phi^{+}, \mathbf{F}_{m} \Psi>} \\
=\frac{<\Phi_{m}^{+}, \mathbf{F}_{n} \Psi>-<\Phi_{m}^{+}, \mathbf{F}_{d n} \Psi>}{\left\langle\Phi_{m}^{+}, \mathbf{F}_{n} \Psi>\right.} \cdot k_{c} \frac{\left\langle\mathbf{F}_{m} \Psi>\cdot<\Phi_{m}^{+}, \mathbf{F}_{n} \Psi>\right.}{<\mathbf{F}_{n} \Psi>\cdot<\Phi^{+}, \mathbf{F}_{m} \Psi>} \cdot\left\langle\mathbf{F}_{n} \Psi>\right. \\
\stackrel{\text { def }}{=}\left(1-\beta_{m n}\right) k_{m n} S_{n}
\end{gathered}
$$

Similarly the left hand side term is developed as: 


$$
\begin{gathered}
k<\Phi_{m}^{+}, \frac{1}{v} \frac{\partial \Psi_{n}}{\partial t}>\frac{\left\langle\mathbf{F}_{m} \Psi\right\rangle}{<\Phi^{+}, \mathbf{F}_{m} \Psi>}=k \frac{\left.<\Phi_{m}^{+}, \frac{1}{v} \frac{\partial \Psi_{n}}{\partial t}\right\rangle}{\frac{d S_{m n}}{d t}} \frac{\left.<\mathbf{F}_{m} \Psi\right\rangle}{\left.<\Phi^{+}, \mathbf{F}_{m} \Psi\right\rangle} \cdot \frac{d S_{m n}}{d t} \\
\stackrel{\text { def }}{=} \ell_{m n} \frac{d S_{m n}}{d t}
\end{gathered}
$$

$\ell_{m n}$ has the dimensionality of time and may be considered an average time needed by neutrons born in region $n$ to reach region $m$ and generate next generation neutrons. The coupled flux-precursor term is developed as:

$$
k<\Phi_{m}^{+}, \chi_{d}^{(i)} C_{n}^{(i)}>\frac{\left\langle\mathbf{F}_{m} \Psi>\right.}{<\Phi^{+}, \mathbf{F}_{m} \Psi>}=k \frac{\left.<\Phi_{m}^{+}, \chi_{d}^{(i)} C_{n}^{(i)}\right\rangle}{\left\langle C_{n}^{(i)}>\right.} \frac{\left\langle\mathbf{F}_{m} \Psi\right\rangle}{\left\langle\Phi^{+}, \mathbf{F}_{m} \Psi>\right.} \cdot\left\langle C_{n}^{(i)}\right\rangle
$$

and written, with notation abuse $\left(C_{n}^{(i)}\right.$ for $\left\langle C_{n}^{(i)}>\right)$ :

$$
<\Phi_{m}^{+}, \chi_{d}^{(i)} C_{n}^{(i)}>\frac{<\mathbf{F}_{m} \Psi>}{<\Phi^{+}, \mathbf{F}_{m} \Psi>} \stackrel{\text { def }}{=} k_{m n}^{(i)} C_{n}^{(i)}
$$

In the precursor equations we define also:

$$
\left\langle v_{d}^{(i)} \Sigma_{f m} \Psi>=\frac{<v_{d}^{(i)} \Sigma_{f m} \Psi>}{\left\langle v \Sigma_{f m} \Psi>\right.} \cdot\left\langle v \Sigma_{f m} \Psi>\stackrel{\text { def }}{=} \beta_{m}^{(i)} S_{m}\right.\right.
$$

Finally, the $N(N+D)$ Avery MPK equations are:

$$
\left\{\begin{array}{c}
\ell_{m n} \frac{d S_{m n}}{d t}=\left(1-\beta_{m n}\right) k_{m n} S_{n}-S_{m n}+\sum_{i=1}^{D} \lambda^{(i)} k_{m n}^{(i)} C_{n}^{(i)} \\
\frac{d C_{m}^{(i)}}{d t}=\beta_{m}^{(i)} S_{m}-\lambda^{(i)} C_{m}^{(i)}
\end{array}\right.
$$

The formal manipulations leading to the sources and coefficients defined in Eq. $(28,29$, $30,32,33)$ involve the unknown kinetic flux $\Psi$. These coefficients are approached using the static flux of the associated critical problem, Eq.(17), and its partial fluxes:

$$
\mathbf{A} \Phi_{m}=\frac{1}{k_{c}} \mathbf{F}_{m} \Phi
$$

We have then:

$$
\begin{aligned}
S_{m n} \approx \frac{\left\langle\mathbf{F}_{m} \Phi><<\Phi^{+}, \mathbf{F}_{m} \Phi_{n}\right\rangle}{<\Phi^{+}, \mathbf{F}_{m} \Phi>} & k_{m n} \approx k_{c} \frac{<\mathbf{F}_{m} \Phi>\cdot\left\langle\Phi_{m}^{+}, \mathbf{F}_{n} \Phi>\right.}{\left\langle\mathbf{F}_{n} \Phi>\cdot<\Phi^{+}, \mathbf{F}_{m} \Phi>\right.} \\
\beta_{m n} \approx \frac{<\Phi_{m}^{+}, \mathbf{F}_{d n} \Phi>}{<\Phi_{m}^{+}, \mathbf{F}_{n} \Phi>} & \beta_{m}^{(i)} \approx \frac{\left\langle v_{d}^{(i)} \Sigma_{f m} \Phi>\right.}{<\mathbf{F}_{m} \Phi>}
\end{aligned}
$$

For the $k_{m n}$ coefficients, through the definition of partial fluxes Eq. $(23,35)$ :

$$
\begin{gathered}
<\Phi_{m}^{+}, \mathbf{F}_{n} \Phi>=k_{c}<\Phi_{m}^{+}, \mathbf{A} \Phi_{n}>=k_{c}<\mathbf{A}^{+} \Phi_{m}^{+}, \Phi_{n}> \\
=<\mathbf{F}_{m}^{+} \Phi^{+}, \Phi_{n}>=<\Phi^{+}, \mathbf{F}_{m} \Phi_{n}>
\end{gathered}
$$

This relation allows falling back on Avery's definition for the $k_{m n}$ (Avery, 1958):

$$
k_{m n} \stackrel{\text { def }}{=} k_{c} \frac{<\mathbf{F}_{m} \Phi>\cdot<\Phi^{+}, \mathbf{F}_{m} \Phi_{n}>}{<\mathbf{F}_{n} \Phi>\cdot<\Phi^{+}, \mathbf{F}_{m} \Phi>}=k_{c} \frac{S_{m n}}{S_{n}}
$$


For the generation times, an asymptotic exponential regime is assumed, where the derivatives are proportional to the functions, and the time eigenfunction is identified with the multiplication factor eigenfunction:

$$
\ell_{m n} \approx k \frac{<\Phi_{m}^{+}, \frac{1}{v} \Phi_{n}>}{S_{m n}} \frac{<\mathbf{F}_{m} \Phi>}{<\Phi^{+}, \mathbf{F}_{m} \Phi>}=k \frac{<\Phi_{m}^{+}, \frac{1}{v} \Phi_{n}>}{\left.<\Phi^{+}, \mathbf{F}_{m} \Phi_{n}\right\rangle}
$$

And finally, the precursor distribution is assumed proportional to the neutron production distribution of the associated critical problem:

$$
k_{m n}^{(i)} \approx k_{c} \frac{<\mathbf{F}_{m} \Phi>\cdot<\Phi_{m}^{+}, \mathbf{F}_{d n}^{(i)} \Phi>}{<\mathbf{F}_{d n}^{(i)} \Phi>\cdot<\Phi^{+}, \mathbf{F}_{m} \Phi>}
$$

\subsection{Formal comparisons of the MPK models}

\subsubsection{Basic features}

Table 1 recapitulates the number of equations, weight functions and approximate formulations for kinetic coefficients based on the calculation of static fluxes.

Table 1 - Multipoint model comparison

\begin{tabular}{|c|c|}
\hline Kobayashi & Avery \\
\hline$N(1+D)$ equations & $N(N+D)$ equations \\
\hline $\begin{array}{c}\text { Weights: } G_{m}^{+} \\
\mathbf{A}^{+} G_{m}^{+}=v \Sigma_{f m}\end{array}$ & Weights: $\Phi_{m}^{+}$ \\
\hline$<G_{m}^{+}, \frac{1}{v} \Phi>$ & $\mathbf{A}^{+} \Phi_{m}^{+}=\frac{1}{k} \mathbf{F}_{m}^{+} \Phi^{+}$ \\
$\ell_{m} \approx \frac{<\mathbf{F}_{m} \Phi>}{<G_{m}^{+}, \mathbf{F}_{d n} \Phi>}$ & $\ell_{m n} \approx k \frac{<\Phi_{m}^{+}, \frac{1}{v} \Phi_{n}>}{<\Phi^{+}, \mathbf{F}_{m} \Phi_{n}>}$ \\
\hline$\beta_{m n} \approx \frac{<G_{m}^{+}, \mathbf{F}_{n} \Phi>}{<G_{m}^{+}, \mathbf{F}_{n} \Phi>}$ & $\beta_{m n} \approx \frac{<\Phi_{m}^{+}, \mathbf{F}_{d} \Phi_{n}>}{<\Phi_{m}^{+}, \mathbf{F} \Phi_{n}>}$ \\
\hline$k_{m n} \approx \mathbf{F}_{n} \Phi>$ & $k_{m n} \approx k \frac{<\mathbf{F}_{m} \Phi>\cdot<\Phi^{+}, \mathbf{F}_{m} \Phi}{<\mathbf{F}_{n} \Phi>\cdot<\Phi^{+}, \mathbf{F}_{m} \Phi>}$ \\
\hline$k_{m n}^{(i)} \approx \frac{<G_{m}^{+}, \mathbf{F}_{d n}^{(i)} \Phi>}{<\mathbf{F}_{d n}^{(i)} \Phi>}$ & $k_{m n}^{(i)} \approx k \frac{<\mathbf{F}_{m} \Phi>\cdot<\Phi_{m}^{+}, \mathbf{F}_{d n}^{(i)} \Phi>}{<\mathbf{F}_{d n}^{(i)} \Phi>\cdot<\Phi^{+}, \mathbf{F}_{m} \Phi>}$ \\
\hline$\beta_{m}^{(i)} \approx \frac{<v_{d}^{(i)} \Sigma_{f m} \Phi>}{<\mathbf{F}_{m} \Phi>}$ & $\beta_{m}^{(i)} \approx \frac{<v_{d}^{(i)} \Sigma_{f m} \Phi>}{<\mathbf{F}_{m} \Phi>}$ \\
\hline
\end{tabular}

\subsubsection{Reduction of Avery's model to Kobayashi's model}

Summing the $n$ Avery flux equations having $m$ as first index yields: 


$$
\sum_{n=1}^{N} \ell_{m n} \frac{d S_{m n}}{d t}=\sum_{n=1}^{N}\left(1-\beta_{m n}\right) k_{m n} S_{n}-S_{m}+\sum_{i=1}^{D} \lambda^{(i)} \sum_{n=1}^{N} k_{m n}^{(i)} C_{n}^{(i)}
$$

This means that we can have a formal identification to Kobayashi's model (at a given moment) if:

$$
\ell_{m} \equiv \frac{\sum_{n=1}^{N} \ell_{m n} \frac{d S_{m n}}{d t}}{\sum_{n=1}^{N} \frac{d S_{m n}}{d t}}
$$

This agrees with the interpretations of $\ell_{m n}$ as the average time needed for neutrons born in region $n$ to reach region $m$ and produce next generation neutrons, and $\ell_{m}$ as the average time for neutrons born in the whole system to reach region $m$ and produce next generation neutrons.

\subsubsection{Reduction of multipoint to point model}

For a single region $(N=1)$, both Kobayashi and Avery sets of equations reduce to:

$$
\left\{\begin{array}{c}
\ell \frac{d S}{d t}=[(1-\beta) k-1] S+\sum_{i=1}^{D} \lambda^{(i)} k^{(i)} C^{(i)} \\
\frac{d C^{(i)}}{d t}=\beta^{(i)} S-\lambda^{(i)} C^{(i)}
\end{array}\right.
$$

If we divide the first equation by $k \ell$ and multiply the precursor equations by $\frac{k^{(i)}}{k \ell}$ we get:

$$
\left\{\begin{array}{c}
\frac{d S}{d t}=\frac{\rho-\beta}{\ell} S+\sum_{i=1}^{D} \lambda^{(i)} \frac{k^{(i)}}{k \ell} C^{(i)} \\
\frac{k^{(i)}}{k \ell} \frac{d C^{(i)}}{d t}=\frac{k^{(i)} \beta^{(i)}}{k \ell} S-\lambda^{(i)} \frac{k^{(i)}}{k \ell} C^{(i)}
\end{array}\right.
$$

Then, defining

$$
\tilde{C}^{(i)}=\frac{k^{(i)}}{k} C^{(i)} \quad \text { and } \quad \tilde{\beta}^{(i)}=\frac{k^{(i)}}{k} \beta^{(i)}
$$

and assuming $\frac{k^{(i)}}{k \ell}$ to be constant, we get:

$$
\left\{\begin{array}{c}
\frac{d S}{d t}=\frac{\rho-\beta}{\ell} S+\sum_{i=1}^{D} \lambda^{(i)} \tilde{C}^{(i)} \\
\frac{d \tilde{C}^{(i)}}{d t}=\frac{\tilde{\beta}^{(i)}}{\ell} S-\lambda^{(i)} \tilde{C}^{(i)}
\end{array}\right.
$$

Which is formally the same equation as Eq.(81) in Appendix. Note that from Eq.(18) or from Eq. $(36,40)$ we can check that $\sum_{i=1}^{D} \widetilde{\beta}^{(i)}=\beta$. However, different choices for the weight function $\left(G^{+}\right.$for Kobayashi, $\Phi^{+}$for Avery and the traditional PK) will make the numerical values of the PK coefficients, even if computed at the same time, slightly different. 


\subsubsection{Prompt jump formulas}

The prompt jump formula can be established readily for the point kinetics model (Eq. (81) in Appendix). Starting from a initial critical state with amplitude $T_{0}$ and equilibrium precursor concentrations such as $\lambda^{(i)} \tilde{C}_{0}^{(i)}=\frac{\widetilde{\beta}^{(i)}}{\ell} T_{0}$, an instantaneous reactivity jump is performed, and the reactivity is kept constant at its new value. If positive, this reactivity insertion is supposed small enough not to reach prompt criticality. Due to the very different timescales of prompt and delayed neutrons $\left(\ell \ll \min _{i} \frac{1}{\lambda^{(i)}}\right)$, we assume to be at a time $t$ such that $\ell \ll t \ll \min _{i} \frac{1}{\lambda^{(i)}}$ and write that the $\tilde{C}^{(i)}$ have not had enough time to change significantly (because $t \ll \min _{i} \frac{1}{\lambda^{(i)}}$ ) but that an equilibrium $T_{p}$ on prompt neutrons has been reached (because $\ell \ll t$ ):

$$
0=\frac{\rho-\beta}{\ell} T_{p}+\sum_{i=1}^{D} \lambda^{(i)} \tilde{C}_{0}^{(i)}=(\rho-\beta) T_{p}+\sum_{i=1}^{D} \tilde{\beta}^{(i)} T_{0}
$$

Hence, the prompt jump formula for point kinetics is:

$$
\frac{T_{p}}{T_{0}}=\frac{\beta}{\beta-\rho}
$$

For the multipoint equations, the derivation proceeds the same way (detail is given only for the Avery equations, but the derivation would be similar for the Kobayashi equations). Starting from a initial critical state with sources $S_{m n}^{0}$ and equilibrium precursor concentrations such as $\beta_{m}^{(i) 0} S_{m}^{0}=\lambda^{(i)} C_{m}^{(i) 0}$, an instantaneous, prompt subcritical, reactivity jump is performed, and the reactivity is kept constant at its new value. We assume again to be at a time $t$ such that $\max _{m, n} \ell_{m n} \ll t \ll \min _{i} \frac{1}{\lambda^{(i)}}$, so that the same assumptions as above hold and:

$$
0=\left(1-\beta_{m n}\right) k_{m n} S_{n}^{p}-S_{m n}^{p}+\sum_{i=1}^{D} k_{m n}^{(i)} \beta_{n}^{(i)} S_{n}^{0}
$$

Here are some definitions to introduce a more compact notation. $S$ is the $N$-vector of generic element $S_{m}$ (with $S_{m}=\sum_{n=1}^{N} S_{m n}$ ), E is the identity $N \times N$ matrix, K the square matrix of generic element $k_{m n}, \mathbf{K}^{+}$its transpose. $\mathbf{K}^{(i)}$ is the square matrix of generic element $k_{m n}^{(i)}$, B the square matrix of generic element $\beta_{m n}, \mathbf{B}^{(i)}$ the square diagonal matrix of generic diagonal element $\beta_{m}^{(i)}$. Finally, $S^{+}$is the fundamental eigenvector of the eigenvalue equation:

$$
\mathbf{K}^{+} S^{+}=k S^{+}
$$

Eq.(48) is multiplied by $S_{m}^{+}$and a summation over $m$ and $n$ is performed; with the notation $\langle\cdot, \cdot\rangle$ for the usual vector dot product we obtain:

$$
0=<S^{+},[\mathbf{K}-(\mathbf{B} \cdot \mathbf{K})-\mathbf{E}] S^{p}>+\sum_{i=1}^{D}<S^{+}, \mathbf{K}^{(i)} \mathbf{B}^{(i)} S^{0}>
$$


where $\mathbf{B} \cdot \mathbf{K}$ represents the entry-for-entry (or Hadamard) product: $(\mathbf{B} \cdot \mathbf{K})_{m n}=$ $B_{m n} K_{m n}$. Then:

$$
\begin{aligned}
& <S^{+}, \mathbf{K} S^{p}>=<\mathbf{K}^{+} S^{+}, S^{p}>=k<S^{+}, S^{p}> \\
& <S^{+}, \mathbf{K} S^{0}>=<\mathbf{K}^{+} S^{+}, S^{0}>=k<S^{+}, S^{0}>
\end{aligned}
$$

We define average values for the global and delayed neutron fractions:

$$
\begin{gathered}
\bar{\beta}=\frac{<S^{+}, \mathbf{B} \cdot \mathbf{K} S^{p}>}{<S^{+}, \mathbf{K} S^{p}>}=\frac{<S^{+}, \mathbf{B} \cdot \mathbf{K} S^{p}>}{k<S^{+}, S^{p}>} \\
\bar{\beta}^{d}=\frac{<S^{+}, \sum_{i=1}^{D} \mathbf{K}^{(i)} \mathbf{B}^{(i)} S^{0}>}{<S^{+}, \mathbf{K} S^{0}>}=\frac{<S^{+}, \sum_{i=1}^{D} \mathbf{K}^{(i)} \mathbf{B}^{(i)} S^{0}>}{k<S^{+}, S^{0}>}
\end{gathered}
$$

so that Eq.(57) can then be written as :

$$
0=(k-\bar{\beta} k-1)<S^{+}, S^{p}>+\bar{\beta}^{d} k<S^{+}, S^{0}>
$$

Dividing by $k$ and rearranging, the prompt jump formula takes a form very similar to Eq.(47):

$$
\frac{<S^{+}, S^{p}>}{<S^{+}, S^{0}>}=\frac{\bar{\beta}^{d}}{\bar{\beta}-\rho}
$$

It now involves weighted amplitudes and delayed neutron fractions.

\section{Numerical schemes to solve multipoint equations}

\subsection{Based on matrix algebra: the MATMPK solver}

The point and multipoint problems involve systems of linear ordinary differential equations and hence can be cast into a vector differential equation:

$$
\frac{d V}{d t}=\mathbf{M}(t) V(t)
$$

The state vector $V$ contains the unknown functions: neutron sources and precursor concentrations (e.g. the $S_{m n}$ and the $C_{m}^{(i)}$ for the Avery equations). Its dimension is $(1+$ $D)$ for PK, $N(1+D)$ for Kobayashi's MPK and $N(N+D)$ for Avery's MPK. The transition matrix $M$ contains the various kinetic coefficients, according to the form of the equations. The formal solution of Eq.(56) over an interval of time $\Delta t$ is:

$$
V(t+\Delta t)=V(t)+\int_{t}^{t+\Delta t} \mathbf{M}(\theta) V(\theta) d \theta
$$

This remains a purely formal solution, as it involves the unknown vector $V(\theta)$. In the specific case when the transition matrix $\mathbf{M}$ is constant, the exact solution is known:

$$
\mathbf{M} \text { constant } \Rightarrow V(t+\Delta t)=\exp (\Delta t \boldsymbol{M}) V(t)
$$

But in the general case, no closed formula is available, and the integral in Eq.(57) has to be approximated to work the problem out numerically. To this end, we shall use here the first simple Newton-Cotes quadrature formulas (see e.g. Abramowitz and Stegun, 1964, $\S 25.4$ ). Table 2 recapitulates the formulas used and their order. To simplify the formulas, we make use of the following notation for all functions and matrices in the "elementary" time interval $[t ; t+\Delta t]: f_{\alpha} \stackrel{\text { def }}{=} f(t+\alpha \Delta t)$. 
Table 2 - The Newton-Cotes quadrature formulas used in this work.

\begin{tabular}{|l|c|}
\hline Rule name & $\int_{t}^{t+\Delta t} \mathbf{M}(\theta) V(\theta) d \theta=\cdots$ \\
\hline Rectangle & $\Delta t \mathbf{M}_{0} V_{0}+0\left(\Delta \mathrm{t}^{2}\right)$ \\
& $\Delta t \mathbf{M}_{1} V_{1}+0\left(\Delta \mathrm{t}^{2}\right)$ \\
\hline Midpoint & $\Delta t \mathbf{M}_{1 / 2} V_{1 / 2}+0\left(\Delta \mathrm{t}^{3}\right)$ \\
Trapezoidal & $\frac{\Delta t}{2}\left(\mathbf{M}_{0} V_{0}+\mathbf{M}_{1} V_{1}\right)+0\left(\Delta \mathrm{t}^{3}\right)$ \\
\hline Simpson & $\frac{\Delta t}{6}\left(\mathbf{M}_{0} V_{0}+4 \mathbf{M}_{1 / 2} V_{1 / 2}+\mathbf{M}_{1} V_{1}\right)+0\left(\Delta \mathrm{t}^{5}\right)$ \\
Newton & $\frac{\Delta t}{8}\left(\mathbf{M}_{0} V_{0}+3 \mathbf{M}_{1 / 3} V_{1 / 3}+3 \mathbf{M}_{2 / 3} V_{2 / 3}+\mathbf{M}_{1} V_{1}\right)+0\left(\Delta \mathrm{t}^{5}\right)$ \\
\hline
\end{tabular}

The formula is said of order $n$ if the order of magnitude of the neglected terms in the formulas above is $\mathrm{O}\left(\Delta \mathrm{t}^{n}\right)$. This is the order for the elementary time interval $[t ; t+\Delta t]$. But what is usually done is to repeat the formula over successive small interval of amplitude $\Delta t$ covering a large time interval $T$; the number of small intervals involved is then $Q=\frac{T}{\Delta t}$, and this generally entails the loss of one order at the global scale: a method of order $n$ on the elementary interval $\Delta t$ is then generally of order $(n-1)$ on the global interval $T=Q \Delta t$ collecting the elementary intervals.

The elementary propagator $\mathbf{P}_{0 \rightarrow 1}$ is defined as the matrix changing $V(t)=V_{0}$ into $V(t+\Delta t)=V_{1}$. If $\mathrm{E}$ is the unit matrix, and using the above notation, the propagator can be expressed as

$$
\mathbf{P}_{0 \rightarrow 1}=\mathbf{E}+\Delta t \int_{0}^{1} \mathbf{M}_{\alpha} V_{\alpha} d \alpha
$$

Eq.(58) gives the exact propagator when $\mathbf{M}$ is constant over time. In the general case, assuming $\mathbf{M}(t)$ is known, either explicitly (prescribed conditions) or iteratively, we shall now approximate this propagator to various orders.

\subsubsection{Order 1: the Euler schemes}

The rectangle rules are used. The left rectangle rule (see Table 2) is explicit and yields the elementary propagator:

$$
\mathbf{P}_{0 \rightarrow 1} \approx \mathbf{E}+\Delta t \mathbf{M}_{0}
$$

This is the explicit Euler method, of global order 1 . However, the problem to be solved is stiff because of the very different timescales involved for prompt and delayed neutrons, and explicit methods are known to behave poorly in such a case: they are unstable except 
for very small (in practice) values of the elementary time step. For example, except if $\Delta t$ is small enough, the dominant eigenvalue of the approximate propagator $\mathbf{E}+\Delta t \mathbf{M}_{0}$ may exceed the dominant eigenvalue of the exact propagator (with same or different sign), resulting in catastrophic divergence after a sufficient number of elementary iterations. This extends to an explicit Taylor expansion of limited order; for example in the simple case when the transition matrix $\mathbf{M}$ is constant, the norm of the generic term $\frac{\Delta t^{n}}{n !} \mathbf{M}^{n}$ in the Taylor expansion of $\exp (\Delta t \mathbf{M})$ may well begin to decrease (not to say be of negligible norm) only after a very large $n$ has been reached.

Implicit schemes are generally much more tolerant about the acceptable elementary intervals $\Delta t$ or may even enjoy unconditional stability. The right rectangle rule (see Table 2) provides the simplest implicit scheme:

$$
\mathbf{P}_{0 \rightarrow 1} \approx\left(\mathbf{E}-\Delta t \mathbf{M}_{1}\right)^{-1}
$$

This is the well-known implicit Euler method, of global order 1. It would remain of order 1 if we replace $\mathbf{M}_{1}$ by a matrix differing from it by a quantity $\mathrm{O}(\Delta \mathrm{t})$, e.g. $\mathbf{M}_{0}$ or $\mathbf{M}_{1 / 2}$. We shall use the latter hereafter:

$$
\mathbf{P}_{0 \rightarrow 1} \approx\left(\mathbf{E}-\Delta t \mathbf{M}_{1 / 2}\right)^{-1}
$$

\subsubsection{Order 2: the Crank-Nicolson scheme}

The trapezoidal rule (see Table 2) is used, and yields the elementary propagator:

$$
\mathbf{P}_{0 \rightarrow 1} \approx\left(\mathbf{E}-\frac{\Delta t}{2} \mathbf{M}_{1}\right)^{-1}\left(\mathbf{E}+\frac{\Delta t}{2} \mathbf{M}_{0}\right)
$$

This is the Crank-Nicolson scheme (seminal paper reproduced in Crank \& Nicolson, 1996), of global order 2. This global order would be unchanged by replacing the trapezoidal rule by the midpoint rule and taking advantage of $V_{1 / 2}=\frac{1}{2}\left(V_{0}+V_{1}\right)+$ $\mathrm{O}\left(\Delta t^{2}\right)$, yielding a more "symmetric" formula for the propagator, still at global order 2 :

$$
\mathbf{P}_{0 \rightarrow 1} \approx\left(\mathbf{E}-\frac{\Delta t}{2} \mathbf{M}_{1 / 2}\right)^{-1}\left(\mathbf{E}+\frac{\Delta t}{2} \mathbf{M}_{1 / 2}\right)
$$

We will make use of the propagator given in Eq.(64) for the order 2 scheme. Besides the two classical propagators Eq.(61a) and Eq.(64) of respective global orders 1 and 2, we now turn on defining higher order propagators.

\subsubsection{Order 3, from Simpson's rule}

Simpson's rule (usually called Simpson's 1/3 rule) states that, on the elementary interval:

$$
V_{1}=V_{0}+\frac{\Delta t}{6}\left(\mathbf{M}_{0} V_{0}+4 \mathbf{M}_{1 / 2} V_{1 / 2}+\mathbf{M}_{1} V_{1}\right)+\mathrm{O}\left(\Delta t^{5}\right)
$$

The local error drops to order 4 if $V_{1 / 2}$ is replaced by its development at local order 3 (backward Crank-Nicolson formula of Eq.(63)): 


$$
V_{1 / 2}=\left[\mathbf{E}+\frac{\Delta t}{4} \mathbf{M}_{1 / 2}\right]^{-1}\left[\mathbf{E}-\frac{\Delta t}{4} \mathbf{M}_{1}\right] V_{1}+0\left(\Delta t^{3}\right)
$$

This results in the following propagator:

$$
\mathbf{P}_{0 \rightarrow 1}=\left(\mathbf{E}-\frac{\Delta t}{6} \mathbf{M}_{1}-\frac{2 \Delta t}{3} \mathbf{M}_{1 / 2}\left[\mathbf{E}+\frac{\Delta t}{4} \mathbf{M}_{1 / 2}\right]^{-1}\left[\mathbf{E}-\frac{\Delta t}{4} \mathbf{M}_{1}\right]\right)^{-1}\left[\mathbf{E}+\frac{\Delta t}{6} \mathbf{M}_{0}\right]
$$

The global error is of order 3 .

\subsection{Order 4, from Newton's rule}

Newton's rule (also called Simpson's 3/8 rule) states that, on the elementary interval:

$$
V_{1}=V_{0}+\frac{\Delta t}{8}\left(\mathbf{M}_{0} V_{0}+3 \mathbf{M}_{1 / 3} V_{1 / 3}+3 \mathbf{M}_{2 / 3} V_{2 / 3}+\mathbf{M}_{1} V_{1}\right)+\mathrm{O}\left(\Delta t^{5}\right)
$$

The local error would remain of order 5 if we could express $V_{1 / 3}$ and $V_{2 / 3}$ at local order 4 from $V_{0}$ or $V_{1}$. This can be done using the previous propagator Eq.(67): the forward propagator from $V_{0}$ to $V_{2 / 3}$ is, at local order 4 :

$$
\mathbf{F}=\left(\mathbf{E}-\frac{\Delta t}{9} \mathbf{M}_{2 / 3}-\frac{4 \Delta t}{9} \mathbf{M}_{1 / 3}\left[\mathbf{E}+\frac{\Delta t}{6} \mathbf{M}_{1 / 3}\right]^{-1}\left[\mathbf{E}-\frac{\Delta t}{6} \mathbf{M}_{2 / 3}\right]\right)^{-1}\left[\mathbf{E}+\frac{\Delta t}{9} \mathbf{M}_{0}\right]
$$

And the backward propagator from $V_{1}$ to $V_{1 / 3}$ is, again at local order 4:

$$
\mathbf{B}=\left(\mathbf{E}+\frac{\Delta t}{9} \mathbf{M}_{1 / 3}+\frac{4 \Delta t}{9} \mathbf{M}_{2 / 3}\left[\mathbf{E}-\frac{\Delta t}{6} \mathbf{M}_{2 / 3}\right]^{-1}\left[\mathbf{E}+\frac{\Delta t}{6} \mathbf{M}_{1 / 3}\right]\right)^{-1}\left[\mathbf{E}-\frac{\Delta t}{9} \mathbf{M}_{1}\right]
$$

Including these expressions into Eq.(68) and rearranging yields:

$$
\mathbf{P}_{0 \rightarrow 1}=\left(\mathbf{E}-\frac{\Delta t}{8} \mathbf{M}_{1}-\frac{3 \Delta t}{8} \mathbf{M}_{1 / 3} \mathbf{B}\right)^{-1}\left(\mathbf{E}+\frac{\Delta t}{8} \mathbf{M}_{0}+\frac{3 \Delta t}{8} \mathbf{M}_{2 / 3} \mathbf{F}\right)
$$

The global error is of order 4 .

We stop here: this kind of formulas for approximated propagators become more and more complex when the global error order increases. Table 3 recapitulates the number of complex matrix calculations (products and inversions) needed at each elementary step. These complex operations may give rise to cumulated round-off errors when increasing the number of elementary intervals, spoiling the asymptotic global order $n$ behaviour for small elementary time intervals.

Table 3 - Complexity of the various schemes

\begin{tabular}{|l|c|c|c|}
\cline { 2 - 4 } \multicolumn{1}{c|}{} & $\begin{array}{c}\text { Global } \\
\text { order }\end{array}$ & $\begin{array}{c}\text { Matrix } \\
\text { products }\end{array}$ & $\begin{array}{c}\text { Matrix } \\
\text { inversions }\end{array}$ \\
\hline Explicit Euler & 1 & 0 & 0 \\
\hline Implicit Euler & 1 & 0 & 1 \\
\hline Crank-Nicolson & 2 & 1 & 1 \\
\hline
\end{tabular}




\begin{tabular}{|l|l|l|l|}
\hline Simpson & 3 & 2 & 2 \\
\hline Newton & 4 & 7 & 5 \\
\hline
\end{tabular}

The free and open source software Scilab (Scilab Enterprises, 2012) has been used to code the previous schemes into a tool called MATMPK. At this stage, with testing purposes in mind, no time step adaptiveness (a single elementary time step, chosen by the user, is used throughout the simulation), no possible remedies to the accumulation of rounding errors and no Richardson-like extrapolation have been envisaged. Scilab is used as a toolbox for matrix algebra operations. The next sections will be devoted to verification studies.

\subsection{Based on stiff ODE solvers: the SACRE code}

The SACRE (이ver for $\underline{\text { Avery's }}$ Coupled $\underline{\text { Reactor }} \underline{\text { Equations) }}$ code has been developed and verified at INL (Palmiotti et al., 2018). It implements and solves a variant of the coupled MPK equations obtained by Avery. Lumped feedbacks or reactivity and temperature types can be accounted for, in order to be able to investigate realistic transient accident scenarios. The main characteristics of the SACRE code are:

- only memory limitations to the numbers: of regions $(N)$, of delayed neutron time families $(D)$, of feedbacks;

- SACRE solves the initial value problem for stiff first-order ODE systems, using a linear multistep method based on backward differentiation formulas and chord iteration with an internally generated (by difference quotient) full Jacobian (Hindmarsh, 1983);

- reactivity variations (external or by feedback) are operated by changes in the coefficients of the coupling matrix $\mathrm{K}$, through linear change or interpolation in "reactivity", i.e. the $1 / k_{i j}$ are assumed to have a piecewise-linear variation in time;

- the parameters for Avery's equations (coupling coefficients and kinetic parameters) are computed externally on a predefined set of configurations and provided as input data; for the studies presented below, they have been computed using a recently developed (Aufiero et al., 2016) version of the Monte-Carlo code SERPENT (Leppänen et al., 2014); achieving low statistical uncertainty on all coefficients requires a large number of neutron histories for complex geometries, specially for coefficients coupling small and/or far away regions (typical order of magnitude: 50 billion histories per configuration).

Note also that SACRE does not solve Eq.(34), but a variant of it, with coupling coefficients for delayed neutrons equal to those for prompt neutrons $\left(k_{m n}^{(i)}=k_{m n}\right)$, region-wise decay constants for delayed neutron precursor families $\lambda_{n}^{(i)}$, and lumped values $\beta_{m n}=\beta_{n}$ (with $\left.\sum_{i=1}^{D} \beta_{n}^{(i)}=\beta_{n}\right)$ : 


$$
\left\{\begin{array}{c}
\ell_{m n} \frac{d S_{m n}}{d t}=\left(1-\beta_{n}\right) k_{m n} S_{n}-S_{m n}+\sum_{i=1}^{D} \lambda_{n}^{(i)} k_{m n} C_{n}^{(i)} \\
\frac{d C_{m}^{(i)}}{d t}=\beta_{m}^{(i)} S_{m}-\lambda_{m}^{(i)} C_{m}^{(i)}
\end{array}\right.
$$

Furthermore, only the coupling coefficients $k_{m n}$ are assumed to change during the transient, all other parameters have constant values.

\section{Verification of MATMPK on point kinetics benchmarks}

Restricting to prescribed reactivity insertions (no feedback), we apply the previous methods to three point kinetics benchmarks (Ganapol, 2013). A point reactor is initially stationary, at zero reactivity, and three kind of reactivity insertion are performed:

- step (4 variants: $-1 /-0.5 /+0.5 /+1 \$$ - objective: amplitude after $100 \mathrm{~s}$ )

- ramp (+0.1\$/s - objective: amplitude after $11 \mathrm{~s})$

- sinusoidal (amplitude 0.675 , period $100 \mathrm{~s}$ - objective: amplitude after $100 \mathrm{~s}$ )

For the step and ramp reactivity insertions, 6 delayed neutron families are used, whereas only one delayed neutron family is used for the sinusoidal reactivity insertion. 10-digit benchmark values are provided for the neutron population (Ganapol, 2013): see Table 4. The objectives quoted above are the most challenging in the benchmarks for the three types of reactivity insertion.

Table 4 - Objectives for the benchmarks (Ganapol, 2013) - initial amplitude value $=1$

\begin{tabular}{|c|c|c|}
\hline Benchmark & Amplitude after... & 10-digit value \\
\hline Step $-1 \$$ & & $2.866764245 \mathrm{E}-02$ \\
$-0.5 \$$ & $100 \mathrm{~s}$ & $7.158285444 \mathrm{E}-02$ \\
$+0.5 \$$ & & $8.006143562 \mathrm{E}+07$ \\
$+1 \$$ & & $2.596484646 \mathrm{E}+89$ \\
\hline Ramp, $0.1 \$ / \mathrm{s}$ & $11 \mathrm{~s}$ & $1.792213607 \mathrm{E}+16$ \\
\hline Sinusoidal & $100 \mathrm{~s}$ & $1.544816514 \mathrm{E}+01$ \\
\hline
\end{tabular}

For the step insertions (constant transition matrix $\mathbf{M}$ ), the exact solution may be obtained separately by matrix exponentiation, a matrix function available in Scilab. The behaviour of the schemes of first to fourth order in MATMPK is illustrated in Fig. 1, showing the relative errors with respect to the presumably exact solution computed by matrix exponentiation. For the ramp and sinusoidal insertions, we use the 10-digit results given in Table 4 as references and plot the relative errors (Fig. 2). Finally, Table 5 collects the CPU times needed per elementary time step. These CPU times have been assessed on the calculations with the finest time steps used, as the global simulation times are here proportional to the number of elementary time steps subdividing the global time interval. All given CPU times are relative to an Intel Xeon CPU E5-2620 v3 @2.40 GHz processor with Gnome 3.14.1 Linux OS. They are obtained using the timer() command in Scilab.

Table 5 - Typical computation times ( $\mu$ s per time step)

Approximate elementary propagator 


\begin{tabular}{|c|c|c|c|c|c|}
\hline Benchmark & $\begin{array}{c}\text { Number of } \\
\text { time steps }\end{array}$ & $\begin{array}{c}\text { Euler } \\
\text { Implicit }\end{array}$ & $\begin{array}{c}\text { Crank- } \\
\text { Nicolson }\end{array}$ & Simpson & Newton \\
\hline Step $\begin{array}{c}-1 \$ \\
-0.5 \$ \\
+0.5 \$ \\
+1 \$\end{array}$ & $10^{6}$ & 56 & 59 & 70 & 113 \\
\cline { 3 - 6 }+1 & & 58 & 60 & 74 & 115 \\
\cline { 3 - 6 } & & 58 & 60 & 73 & 116 \\
\hline Ramp, 0.1 \$/s & $1.110^{6}$ & 151 & 154 & 261 & 420 \\
\hline Sinusoidal & $10^{6}$ & 84 & 87 & 125 & 185 \\
\hline
\end{tabular}
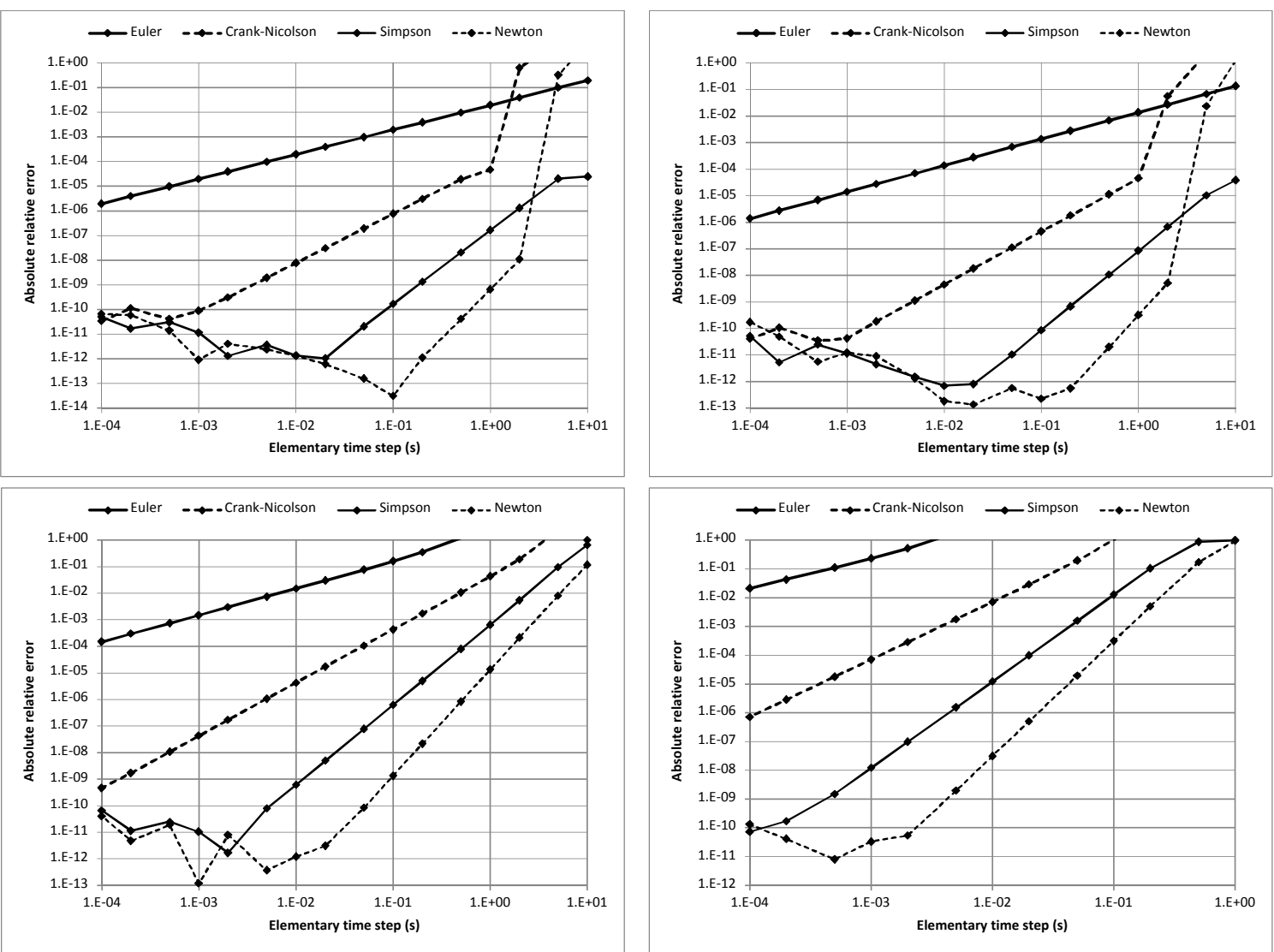

Fig. 1. - Relative errors with respect to exact (double precision) solution as functions of time step size for the four step insertions: -1 (upper left), $-0.5 \$$ (upper right), $+0.5 \$$ (lower left), $+1 \$$ (lower right), and the four approximate propagators. 

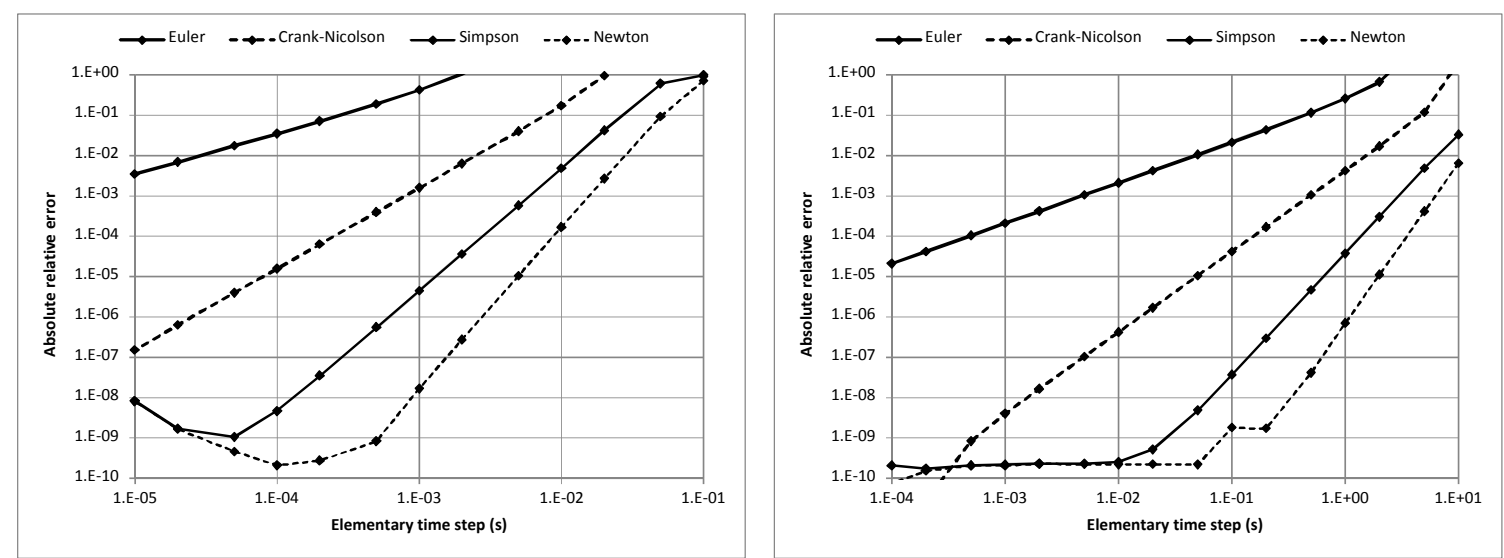

Fig. 2. - Relative errors with respect to the 10-digit reference solution (Table 4) as functions of time step size for the ramp insertion (left) end the sinusoidal insertion (right).

The log-log plots in Fig.1 and Fig.2 show directly, and confirm, the integer slopes corresponding to orders 1 to 4 . There may be a transient adaptation for large elementary time intervals. On the other hand, saturation is reached for very small elementary time intervals for the higher order methods (orders 2, 3 and 4); this is presumably due to the accumulation of round-off errors. Scilab operates in double precision (i.e. on approx. 16 digits); it is expected that for a tool working in quadruple precision, this saturation would be postponed to much smaller relative errors. Nevertheless, the methods of order 3 and 4 allow reaching very good precision (say 5 to 8 exact digits) with a very limited expense of calculation time. Calculation time may not be a problem for PK equations with $(1+D)$ unknown functions, see Table 5, but could become one for MPK problems, with $N(1+D)$ unknown functions (Kobayashi), and even more with $N(N+D)$ unknown functions (Avery), when increasing the number $N$ of physically meaningful regions used.

\section{Simple multipoint MATMPK verification tests}

\subsection{A fast reactor core 3-region model}

We use an early sodium-cooled fast reactor ASTRID CFV core design (Varaine et al., 2011), see Fig.3. A very similar model has been used also for recent SACRE studies (Palmiotti et al., 2018). The core, having a $2 \pi / 3$ rotational symmetry with control rod banks at the same insertion level, is divided in three regions respecting this rotational symmetry and also pictured in Fig. 3. In this simple core division, devised for testing purposes only, each region includes both inner and outer fuel subassemblies. The circled rod subassembly in Fig.3 is the one moving in the ramp reactivity transient studied below.

\subsection{The calculation of MPK coefficients}

These coefficients are computed using the ERANOS code system (Ruggieri et al., 2006). Fluxes and weight functions are computed in diffusion theory with 33 energy groups, using JEFF-3.1.1 data (Santamarina et al., 2009) and delayed neutron data in $D=8$ time families. The various coefficients needed for the MPK equations are computed using the 
perturbation theory capabilities of the code and according to the formulas collected in Table 1. Calculation is automatized by means of the user's language of ERANOS.

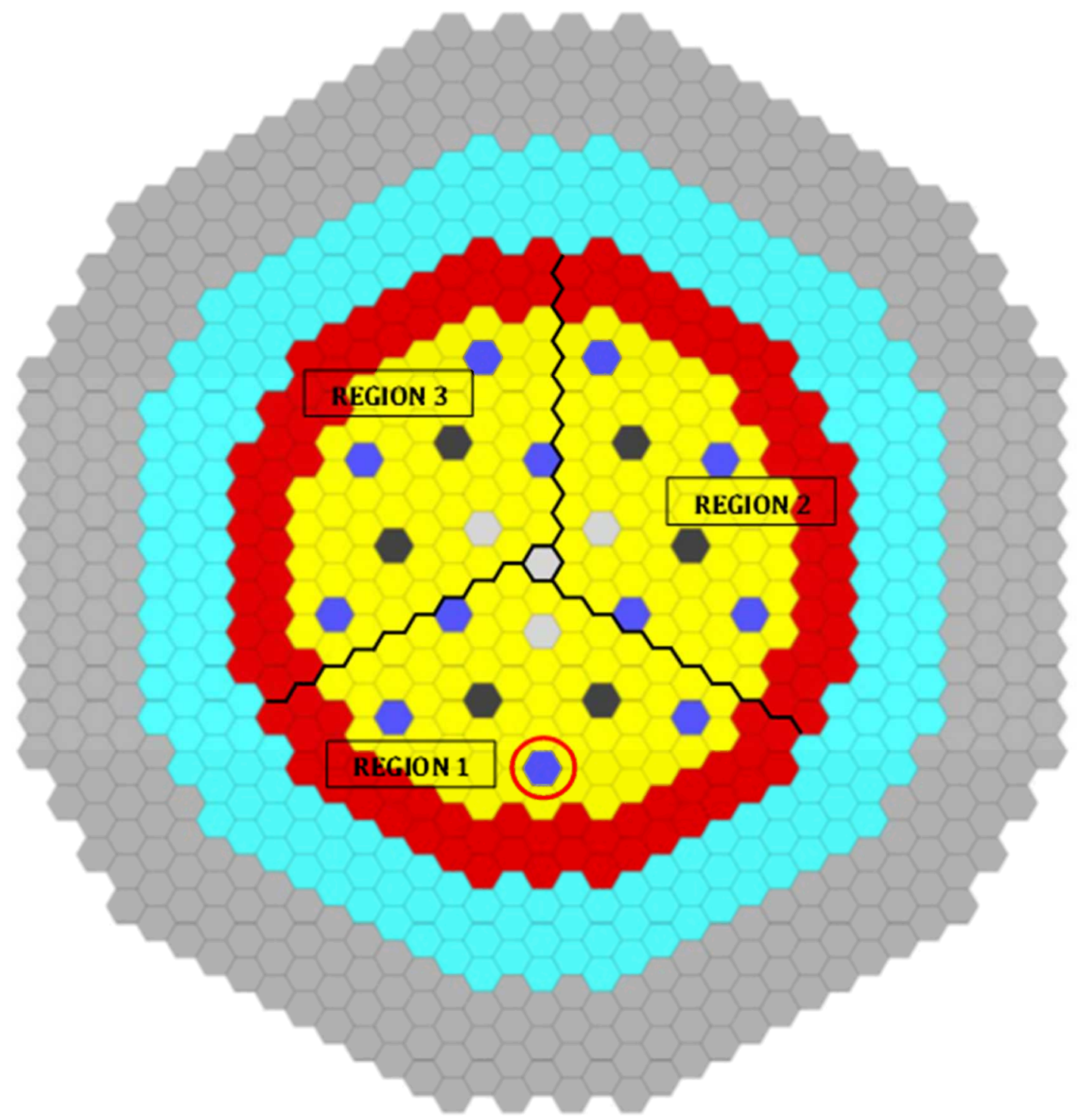

Fig. 3. - Na-cooled fast reactor core used (ASTRID), its division into 3 regions and (circled) the control rod position used for the rod withdrawal transient. Yellow $=$ inner core; red = outer core; light blue $=$ steel-based reflector; grey $=$ radial shielding; dark blue and black = control and shutdown rods; white $=$ diluent rods.

As an illustration, examples of multiplication factors, coefficients and static MPK distributions (direct and adjoint) for the reference case (labelled 0) and the case when the circled control rod in Fig. 3 is raised by $35 \mathrm{~cm}$ (labelled 1) are given respectively in Table 6 and Table 7. It can be observed that, for the reference core with $2 \pi / 3$ rotational symmetry, the coupling matrix reflects this rotational symmetry, i.e. $k_{11}=k_{22}=k_{33}$, $k_{12}=k_{23}=k_{31}$ and $k_{21}=k_{32}=k_{13}$, but with small differences $\left(k_{12} \neq k_{21}\right)$ as the boundaries used for splitting the core into three regions are not invariant under the mirror symmetries of the real core (e.g. the mirror symmetry with respect to the "vertical" line through core centre in Fig. 3). 
The coupling coefficients, as constructed, respect the neutron balance in the core; this means that the fundamental eigenvalue $(k)$ and fission source distribution $(S)$ are the same when computed from the coupling matrix $\mathrm{K}$ built indifferently from Kobayashi or Avery coefficients, and identical to the values computed on the full core model. However, this has no reason to extend to the fundamental adjoint source distribution $\left(S^{+}\right)$of the adjoint (transpose) matrix $\mathbf{K}^{+}$, except if symmetries impose the same distribution, as in the reference core.

The $\ell_{m n}$ in the Avery model show that neutrons take more time to travel between distant regions than within a single region (e.g. here $\ell_{12} \gg \ell_{11}$ ); however, as only a small proportion of neutrons born in a region give rise to next generation in another region (e.g. here $k_{12} \ll k_{11}$ ), we have $\ell(P K) \approx \ell_{11} \approx \ell_{22} \approx \ell_{33}$.

Table 6 - Some of the MPK coefficients (reference core). PK integral values: $k_{0}=$ $1.00313, \beta_{0}=375.2 \mathrm{pcm}, \ell_{0}=0.3801 \mu \mathrm{s}$.

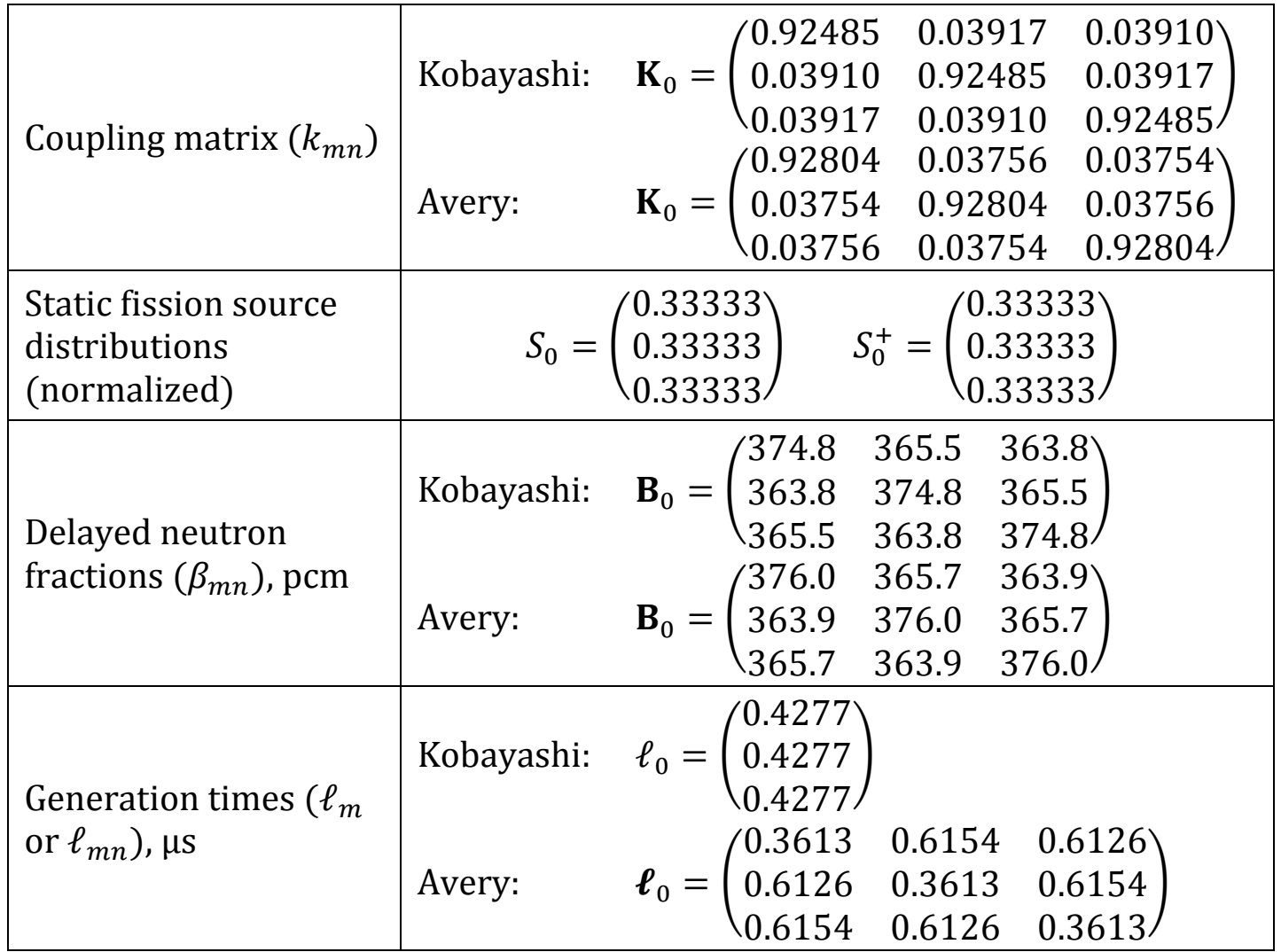

Table 7 - Some of the MPK coefficients (control rod lifted by $35 \mathrm{~cm}$ ). PK integral values: $k_{1}=1.00616, \beta_{1}=375.3 \mathrm{pcm}, \ell_{1}=0.3815 \mu \mathrm{s}$.

\begin{tabular}{|l|lr}
\hline \multirow{3}{*}{ Coupling matrix $\left(k_{m n}\right)$} & & \\
& Kobayashi: & $\mathbf{K}_{1}=\left(\begin{array}{lll}0.94152 & 0.04438 & 0.04351 \\
0.03524 & 0.92123 & 0.03798 \\
0.03402 & 0.03743 & 0.92150\end{array}\right)$ \\
& \multirow{2}{*}{ Avery: } & $\mathbf{K}_{1}=\left(\begin{array}{lll}0.95226 & 0.03766 & 0.03561 \\
0.03820 & 0.92029 & 0.03489 \\
0.03616 & 0.03488 & 0.92116\end{array}\right)$ \\
\hline
\end{tabular}




\begin{tabular}{|c|c|c|c|c|}
\hline $\begin{array}{l}\text { Static fission source } \\
\text { distributions } \\
\text { (normalized) }\end{array}$ & $\begin{array}{l}\text { Kobayashi } \\
\text { Avery }\end{array}$ & $\begin{array}{l}S_{1}= \\
S_{1}=\end{array}$ & $\begin{array}{l}0.40472 \\
0.30000 \\
0.29528 \\
0.40472 \\
0.30000 \\
0.29528\end{array}$ & $\begin{array}{l}S_{1}^{+}=\left(\begin{array}{l}0.34886 \\
0.32574 \\
0.32540\end{array}\right) \\
S_{1}^{+}=\left(\begin{array}{l}0.40828 \\
0.29827 \\
0.29344\end{array}\right)\end{array}$ \\
\hline $\begin{array}{l}\text { Delayed neutron } \\
\text { fractions }\left(\beta_{m n}\right), \mathrm{pcm}\end{array}$ & $\begin{array}{l}\text { Kobayashi: } \\
\text { Avery: }\end{array}$ & $\begin{array}{l}\mathbf{B}_{1}= \\
\mathbf{B}_{1}=\end{array}$ & $\begin{array}{l}\left(\begin{array}{l}375.2 \\
362.6 \\
364.3\end{array}\right. \\
\left(\begin{array}{l}376.6 \\
363.2 \\
365.1\end{array}\right. \\
\end{array}$ & $\begin{array}{ll}365.7 & 364.1 \\
374.5 & 364.9 \\
363.3 & 374.5 \\
365.1 & 363.3 \\
375.6 & 364.9 \\
363.2 & 375.6\end{array}$ \\
\hline $\begin{array}{l}\text { Generation times }\left(\ell_{m}\right. \\
\left.\text { or } \ell_{m n}\right), \mu \mathrm{s}\end{array}$ & Kobayashi: & $\begin{array}{l}\ell_{1}= \\
\ell_{1}=\end{array}$ & $\begin{array}{l}\left(\begin{array}{l}0.4317 \\
0.4291 \\
0.4293\end{array}\right) \\
\left(\begin{array}{l}0.3690 \\
0.6115 \\
0.6162\end{array}\right. \\
\end{array}$ & $\left.\begin{array}{ll}0.6161 & 0.6145 \\
0.3588 & 0.6214 \\
0.6178 & 0.3595\end{array}\right)$ \\
\hline
\end{tabular}

\subsection{Verification : step insertion test}

We assume the core to be critical in the reference state, i.e. the coupling matrices $\mathbf{K}_{0}$ and $\mathbf{K}_{1}$ are divided by the dominant eigenvalue of the reference state $\left(k_{0}\right)$. Starting from a stable reference state, the control rod circled in Fig. 3 is instantaneously lifted by $35 \mathrm{~cm}$, inserting a reactivity $\rho=0.00301<\beta$. The transient is studied from 0 to 1 second. For this step insertion, the exact final value can be computed by matrix exponentiation. A PK step insertion of the same amount of reactivity is also simulated for comparison. The convergence behaviour of the 4 propagators used is given in Fig. 4 (absolute value of the relative error on the global amplitude with respect to the exact solution vs. elementary time step size). For this transient, the propagators of odd order (implicit Euler and Simpson) behave better than the even order ones (Crank-Nicolson and Newton).
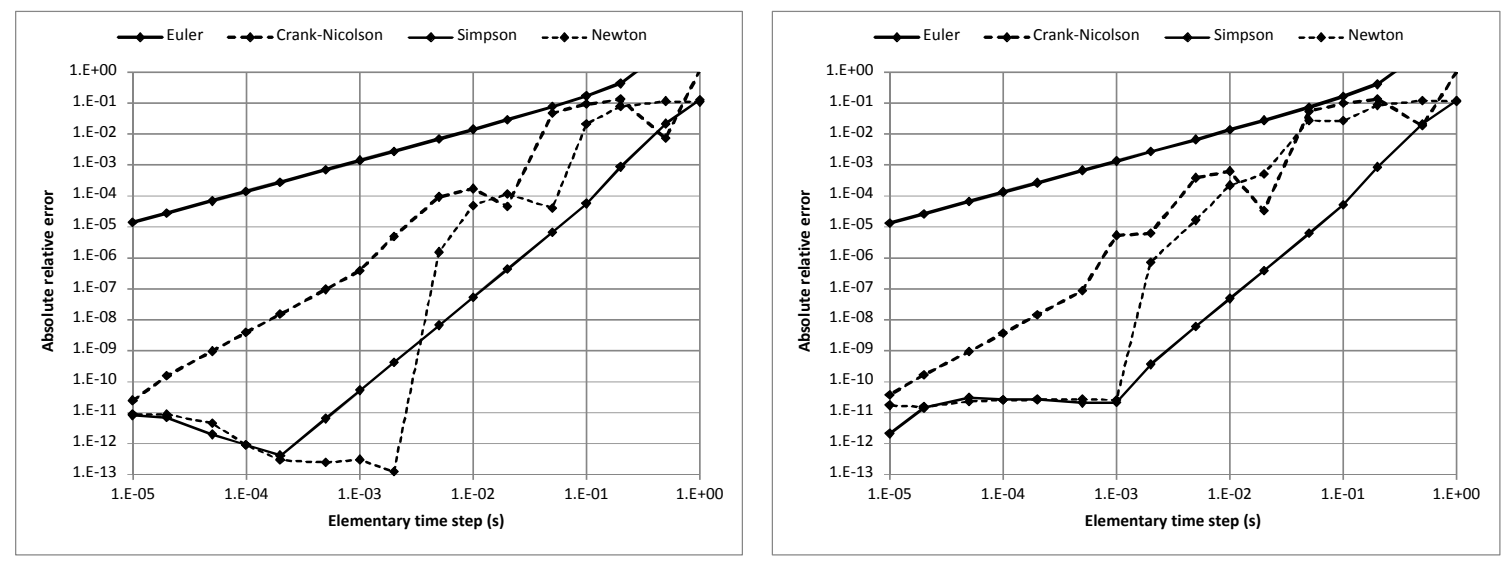

Fig. 4. - 3-point MPK core test: step insertion. Kobayashi formulation (left), Avery formulation (right) 
Computation times (Table 8) increase with the size of the problem, even if here, with $N=$ 3 and $D=8$, the sizes of the matrices involved remain modest: $9 \times 9(\mathrm{PK}), 27 \times 27(\mathrm{MPK}$, Kobayashi) and $33 \times 33$ (MPK, Avery). Anyway, values exact to 5-8 places can be obtained using the Simpson propagator with a limited number of time steps, resulting in very short computation times $(<1$ s).

Table 8 - 3-point MPK core tests: computation times ( $\mu$ s per time step) for the finest time steps used

\begin{tabular}{|ll|c|c|c|c|}
\cline { 3 - 6 } \multicolumn{1}{c|}{} & \multicolumn{4}{c|}{ Approximate elementary propagator } \\
\hline Case & $\begin{array}{c}\text { Euler } \\
\text { Implicit }\end{array}$ & $\begin{array}{c}\text { Crank- } \\
\text { Nicolson }\end{array}$ & Simpson & Newton \\
\hline Step & PK & 56 & 59 & 72 & 112 \\
& MPK (Kobayashi) & 125 & 150 & 280 & 638 \\
& MPK (Avery) & 167 & 196 & 355 & 954 \\
\hline Ramp PK & 151 & 154 & 266 & 405 \\
MPK (Kobayashi) & 1470 & 1510 & 2997 & 4738 \\
MPK (Avery) & 4615 & 4632 & 9321 & 14550 \\
\hline
\end{tabular}

Starting from 1 at $t=0$, the amplitudes $\left(=\sum_{m=1}^{N} S_{m}\right)$ reached at $t=1 \mathrm{~s}$ are very similar; their 5-digit rounded values are 31.996 (PK), 33.483 (MPK, Kobayashi) and 31.317 (MPK, Avery). The time constant for the prompt jump in amplitude is (PK) $\frac{\ell}{\rho-\beta} \approx-5.110^{-4} \mathrm{~s}$ for a jump by a factor $\frac{\beta}{\beta-\rho} \approx 5.1$; this is reflected by the plot given in Fig. 5 (left). The relaxation time for the fission source distribution adjustment to the new asymmetric asymptotic distribution is expected to be of the order of a few generation times $(\ell \approx 0.4$ $\mu \mathrm{s}$ ), the asymptotic shape being close to the static fission source distribution (see $S_{1}$ in Table 7). This is also confirmed by Fig. 5 (right).
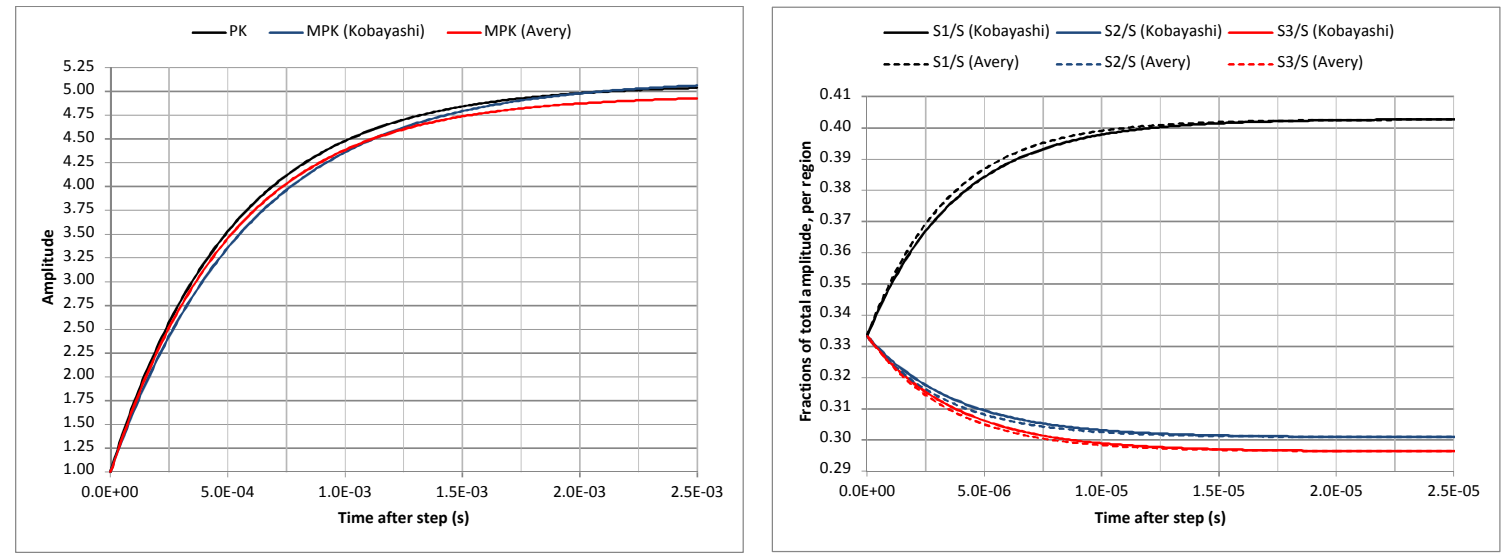

Fig. 5. - 3-point MPK core test: step insertion. Prompt jump in amplitude (left) and shape adjustment (right) of the fission source distribution.

\subsection{Application to a reactivity ramp test}


Here, starting from a stable reference state, the control rod circled in Fig. 3 is progressively lifted by $35 \mathrm{~cm}$, inserting a reactivity $\rho=0.00301<\beta$. The transient is studied from 0 to 1 second. In each of the three variants (PK, Kobayashi-MPK and AveryMPK) all kinetic parameters and coupling coefficients are assumed to vary linearly with time between their initial and final values. This means that the transients do not represent the same physical scenario anymore, as for example the variations in time of the multiplication factor are no more the same. For the PK transient, the multiplication factor $k$ varies linearly in time; for the MPK transients it is the coupling matrix $\mathrm{K}$ that varies linearly in time, and no more the multiplication factor (the dominant eigenvalue of K). The variation laws of the multiplication factor are plotted in Fig. 6.

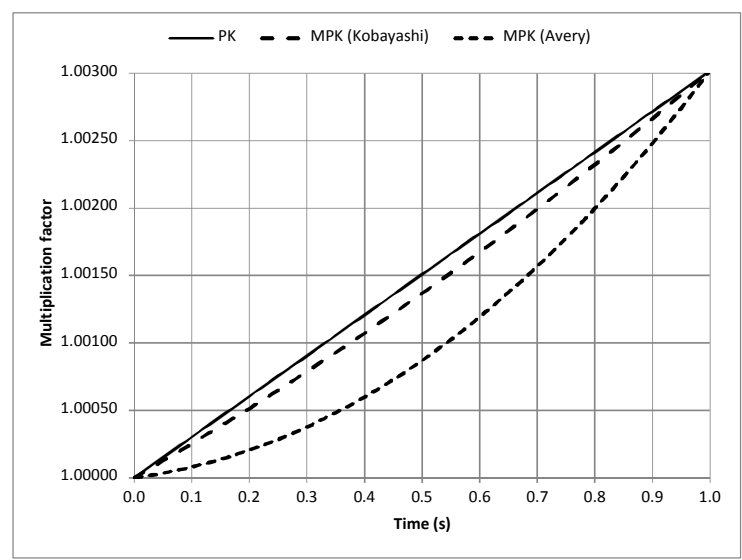

Fig. 6. - Variation law of the multiplication factor in the ramp tests (PK and 3-point MPK with Kobayashi's and Avery's models)

The global reactivity change from $t=0$ to $t=1 \mathrm{~s}$ is given by the exact perturbation formula $\Delta k_{e x}=\frac{\left\langle S_{0}^{+}, \Delta K S_{1}\right\rangle}{\left\langle S_{0}^{+}, S_{1}\right\rangle} \approx 0.00302$, whereas the first-order perturbation formula, giving the slope of the $k(t)$ curve at $t=0$ is $\Delta k_{1 o}=\frac{\left\langle S_{0}^{+}, \Delta K S_{0}\right\rangle}{\left\langle s_{0}^{+}, s_{0}\right\rangle}$; this gives $\Delta k_{1 o} \approx 0.00247$ with the Kobayashi $\mathrm{K}$ matrices, but only $\Delta k_{1 o} \approx 0.00057$ with the Avery K matrices.

The convergence behaviour of the propagators used is given in Fig. 7. Having no external benchmark solution here, the reference used to compute the relative error is a Richardson extrapolated value for the Simpson propagator obtained for $\Delta t \in$ $\left[10^{-4} ; 10^{-3}\right]$, as in this elementary time step interval an asymptotic order 3 for the Simpson propagator seems to be reached (see Fig. 7). For the Simpson and Newton propagators, the asymptotic order behaviour (order 3 or 4 ) is reached only for small elementary time steps and is at least partially masked by its subsequent degradation due to round-off error accumulation. 

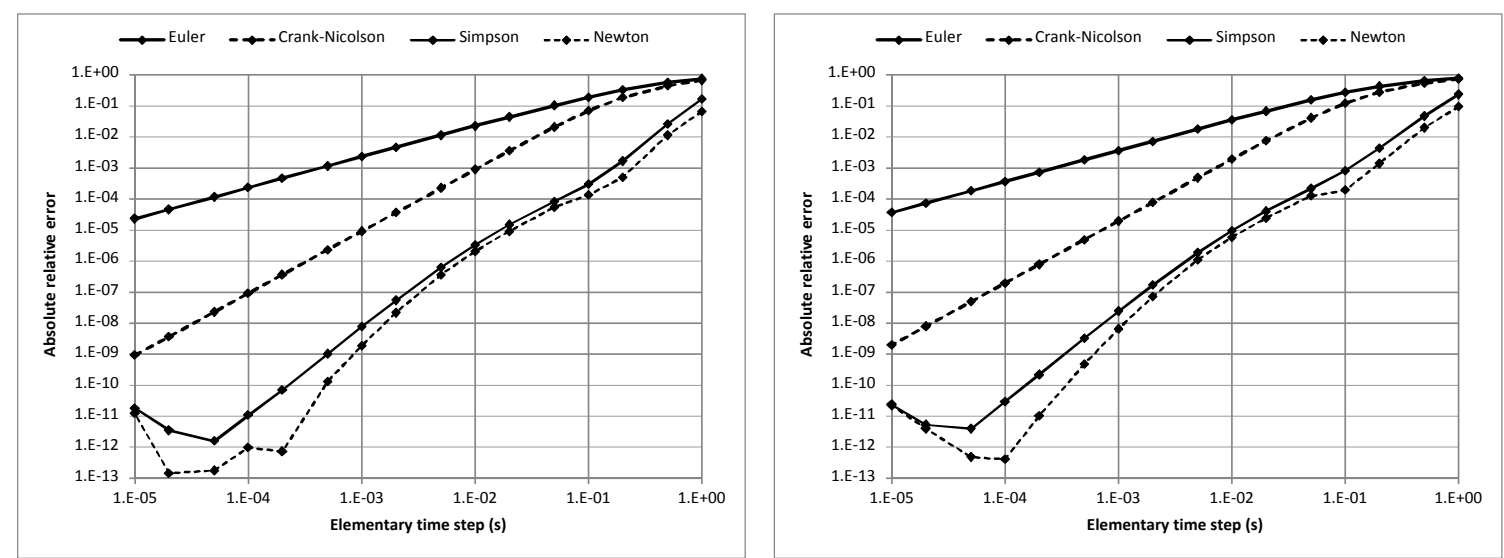

Fig. 7. - 3-point MPK core test: ramp insertion. Kobayashi formulation (left), Avery formulation (right)

Computation times per elementary time step are also given in Table 8. They increase noticeably with respect to the step reactivity insertion cases. However, the only difference between the step and the ramp cases is that in the step cases, the transition matrix step $\mathbf{M}$ is computed only once, whereas for the ramp cases $\mathbf{M}$ has to be computed 1 (Euler implicit and Crank-Nicolson), 2 (Simpson) or 3 (Newton) times per elementary time step. It is then supposed that the interpreted (slow) part of the code script, mainly devoted to fill in the matrix $\mathbf{M}$ at various time steps is the bottleneck with respect to computation time, as opposed to the compiled (fast) part devoted to matrix algebra, i.e. here mainly matrix products and inversions. Anyway, good accuracy (at least 5 digits on the final amplitude) can be obtained still with the Simpson or Newton propagators at limited time expense $(<1 s)$.

Amplitudes and shapes are plotted in Fig. 8 and qualitatively match expectations: for final amplitudes, Avery MPK < Kobayashi MPK $<$ PK due to the time law of reactivity insertion shown in Fig. 6; and there is a continuous shape adjustment to values close to the dominant eigenfunction of the current coupling matrix $\mathbf{K}(t)$, due to very small generation times.
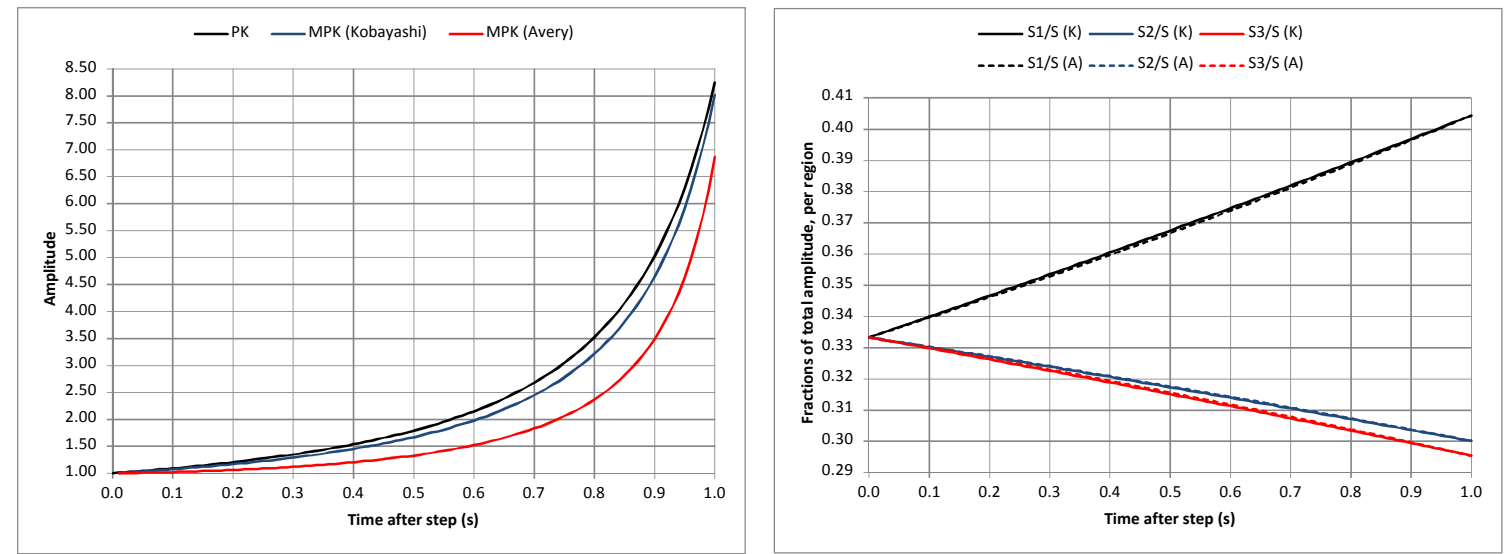

Fig. 8. - 3-point MPK core test: ramp insertion. Progressive adjustment in amplitude (left) and shape (right) of the fission source distribution.

\section{Verification checks with SACRE}


The verification in Section 5.4, although satisfactory, is a partial one, as it lacks numerical comparison against exact solution or results from other MPK solvers. We have chosen to check MATMPK against the existing SACRE code on a simple MPK transient, taking advantage of the fact that SACRE calculations on transients similar to those studied in Section 5.4 (PK + MPK reactivity ramp in an ASTRID-like core divided in three azimuthal regions) have been performed already (Palmiotti et al., 2018). We reproduce one of them with MATMPK; Eq.(72) is solved, with the exception that, as coded in MATMPK, only one set of delayed neutron time constants $\lambda^{(i)}$ with $D=6$ delayed neutron time families is used (in this core, there is a very small region-wise variation for these parameters).

The transient modelled is the progressive extraction of a single rod (analogous to the extraction of the circled rod in Fig. 3). The PK model for this transient assumes a linear insertion of $713 \mathrm{pcm}$ in 8.5 seconds, i.e. a constant reactivity insertion speed of $\approx 84$ $\mathrm{pcm} / \mathrm{s}$, with no feedback (a possible feedback could be an emergency scram). In the MPK model for this transient, the core is divided in three azimuthal regions as in Fig. 3, the coupling matrix $\mathrm{K}$ is computed for the rod in and the rod out states, and is interpolated this way:

$$
\frac{1}{k_{m n}(t)}=\frac{1}{k_{m n}^{\text {rod_in }}}+\frac{t}{8.5}\left(\frac{1}{k_{m n}^{\text {rod_out }}}-\frac{1}{k_{m n}^{\text {rod_in }}}\right)
$$

This interpolation method is similar, for each coupling coefficient $k_{m n}$, to the linear reactivity interpolation in the PK transient, as in PK this means a linear variation for the inverse of the multiplication factor.

Table 9 collects some results obtained after 2 seconds of reactivity insertion. At the beginning of the transient $(t=0)$, the global amplitude is set to one, and the relative amplitudes in the almost symmetric initial core are very close to $1 / 3$ each. The agreement between SACRE and MATMPK is excellent, but one question arises: why does the MPK transient, meant to represent a similar reactivity insertion as the PK transient, result in so different amplitudes after $t=2$ seconds? This question was already raised by the results presented in (Palmiotti et al., 2018) where, depending on the choice of the shape and number of regions, modelling - presumably - the same transient ended up in very different time behaviours for the total amplitude.

The answer has already been given incidentally in Fig. 6 above: a given variation law for the coupling coefficients $k_{m n}$ does not imply the same variation law for the dominant eigenvalue $k$ of the resulting time dependent coupling matrix $\mathrm{K}: k$, being the dominant solution of the $(N+1)^{t h}$-degree algebraic equation $\operatorname{det}(\kappa \mathbf{E}-\mathbf{K})=0$, is a non-linear function of the coupling coefficients $k_{m n}$. We have the same qualitative behaviour as in Fig. 6 here; Fig. 9 gives the quantitative picture of it in the interval from 0 to 2 seconds. An obvious remediation for this discrepancy would be to perform the calculation of the coupling matrix over points in a time grid tight enough to make the interpolated reactivity variations within the time grid intervals almost independent from the model used (PK, MPK with various region patterns). 
Table 9 - Comparison MATMPK/SACRE on PK and MPK transients. Amplitude (global source $S$ ) at $t=2$ second; for the MPK transient the relative region-wise sources $s_{m}=$ $\frac{s_{m}}{S}$ are given also, at $t=2$ second.

\begin{tabular}{|c|c|c|}
\cline { 2 - 3 } \multicolumn{1}{c|}{} & SACRE & MATMPK \\
\hline PK & $S=2.51183$ & $S=2.51173$ \\
\hline $\begin{array}{c}\text { MPK, 3 azimuthal } \\
\text { regions }\end{array}$ & $S=1.23593$ & $S=1.23680$ \\
\cline { 2 - 3 } & $\left\{\begin{array}{l}s_{1}=0.329744 \\
s_{2}=0.329121 \\
s_{3}=0.341137\end{array}\right.$ & $\begin{array}{l}s_{1}=0.329738 \\
s_{2}=0.329110 \\
s_{3}=0.341152\end{array}$ \\
\hline
\end{tabular}

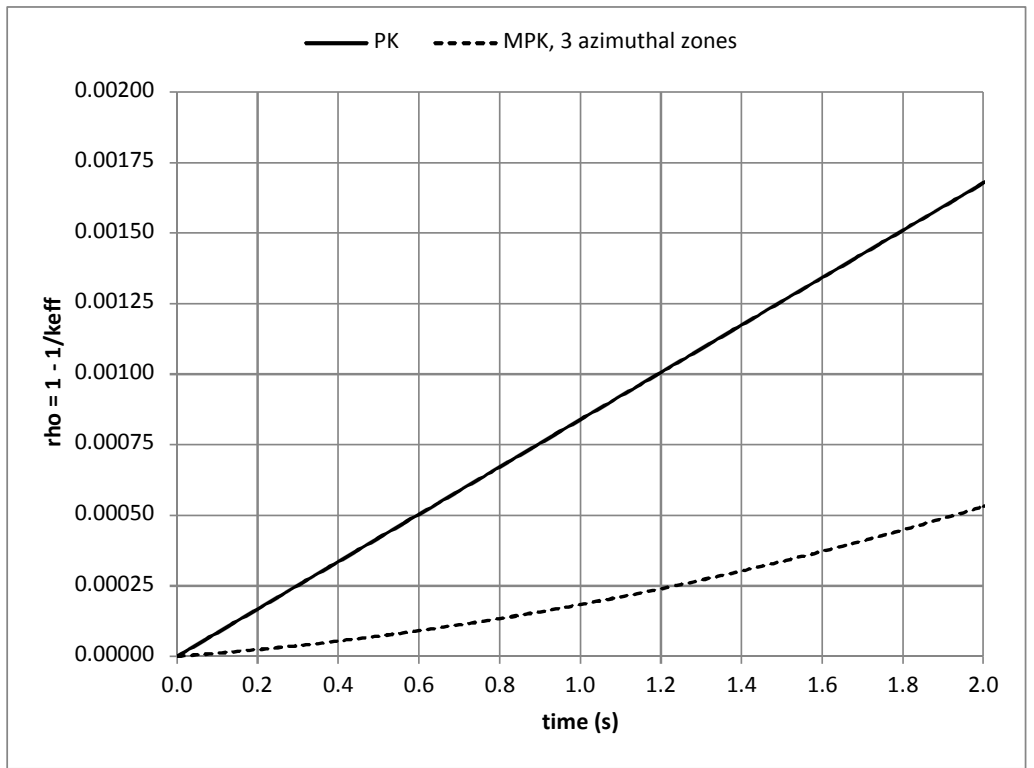

Fig. 9. - Reactivity inserted as a function of time in the PK and MPK transients used for the SACRE/MATMPK comparisons. For the 3-region transient, reactivity is computed as $\rho(t)=1-\frac{1}{k_{e f f}(t)}$, where $k_{e f f}(t)$ is the dominant eigenvalue of the interpolated coupling $\operatorname{matrix} \mathbf{K}(t)$.

\section{Conclusion}

After recalling the derivation of Kobayashi's and Avery's sets of multipoint kinetic equations, these equation systems have been solved numerically by using matrix algebra software and $1^{\text {st }}$ to $4^{\text {th }}$ order implicit schemes based on single-step matrix propagators. The resulting tool, named MATMPK, has been verified on demanding point kinetics benchmarks (10-digit benchmark values, large amplitude variations) with various prescribed reactivity insertions. At this stage, no refinements such as adaptive time step size or extrapolation have been used but, even then, the 10-digits objective can be met easily using the higher order propagators (orders 3 and 4). "Practical" accuracies (say in the 5-digit range) can be obtained with these $3^{\text {rd }}$ or $4^{\text {th }}$ order propagators using a limited number of time steps and calculation time. However, the accumulation of round-off errors prevents reaching relative accuracies much tighter that $10^{-10}$ for very small time step sizes with the simple schemes and double precision software used. 
As a next simple verification test, a 3-region fast reactor core model was built and the coefficients involved in the multipoint kinetics equations computed using the ERANOS code system for i) a reference symmetric state with all control rods inserted at the same height and ii) an asymmetric configuration corresponding to the extraction of a single control rod. Step and ramp reactivity insertions were modelled with MATMPK and the results checked successfully against exact analytical solution (step insertion) or physical intuition (ramp insertion). A final check of MATMPK has been performed, on a ramp insertion, against the existing SACRE MPK code developed at INL and the results produced by the two codes were found in excellent agreement, on amplitude and shape. Usually, MPK coefficients are computed on a discrete set of points in time and interpolated in between; it has been shown that care must be exerted to ensure that this grid of time points is tight enough for the interpolated values to remain representative of those obtained asymptotically for an extremely tight time grid.

Globally, these are promising results and prospects for future improvement in MATMPK are: refined resolution strategies such as adaptiveness criteria for non-uniform time step sizes and Richardson-like extrapolation methods, shift to a fully compiled code to reduce computation times when dealing with "large" numbers of fissile regions and/or feedback. On the longer term, a versatile, fast and accurate multipoint kinetics capability will prove useful for zero-power reactor experiment design, specially in the case of fast-thermal coupled systems (as was the initial motivation for Avery's coupled reactors theory), but also possibly for inclusion in quasi-static space-time kinetics methods, aiming at adaptive shape time step prediction and region-wise level adjustment for the flux initialization of shape calculations.

\section{Acknowledgements}

One of the authors of this submitted manuscript, G. Palmiotti, performed this work as a contractor of the U.S. Government under DOE Contract No. DE-AC07-05ID14517.

\section{Appendix - Derivation of the PK model}

A single, time-independent, weight function $W$ is used and the "amplitude" $S$ is defined as the weighted neutron population for the whole reactor:

$$
S=\left\langle W, \frac{1}{v} \Psi\right\rangle
$$

When looking at variations starting from and remaining close to an initial critical state, the usual choice for the weight function is an adjoint static flux $\Phi_{0}^{+}(\vec{r}, E, \vec{\Omega})$ for this initial critical state, i.e. a solution of:

$$
\left(\mathbf{F}_{0}^{+}-\mathbf{A}_{0}^{+}\right) \Phi_{0}^{+}=0
$$

We also define the forward static flux $\Phi_{0}(\vec{r}, E, \vec{\Omega})$ for the initial critical state, solution of:

$$
\left(\mathbf{F}_{0}-\mathbf{A}_{0}\right) \Phi_{0}=0
$$


Eq.(74) and Eq.(75) are homogeneous and their solutions need a normalization condition to be unequivocally defined. For example, $\Phi_{0}$ may be normalized to the initial reactor power and then $\Phi_{0}^{+}$normalized so that the initial amplitude is one:

$$
S_{0}=<\Phi_{0}^{+}, \frac{1}{v} \Phi_{0}>=1
$$

We can then write in the right-hand side of the flux equation in Eq. (8):

$$
\begin{gathered}
<\Phi_{0}^{+},\left(\mathbf{F}_{p}-\mathbf{A}\right) \Psi>=\left(\frac{\left\langle\Phi_{0}^{+},(\mathbf{F}-\mathbf{A}) \Psi>\right.}{<\Phi_{0}^{+}, \mathbf{F} \Psi>}-\frac{\left\langle\Phi_{0}^{+}, \mathbf{F}_{d} \Psi>\right.}{\left\langle\Phi_{0}^{+}, \mathbf{F} \Psi>\right.}\right) \frac{\left.<\Phi_{0}^{+}, \mathbf{F} \Psi\right\rangle}{\left\langle\Phi_{0}^{+}, \frac{1}{v} \Psi>\right.} S \\
\stackrel{\text { def }}{=} \frac{\rho-\beta}{\ell} S
\end{gathered}
$$

where we have defined the kinetic multiplication factor $(k)$, reactivity $(\rho)$, delayed neutron fraction $(\beta)$ and generation time $(\ell)$ as:

$$
k=\frac{\left\langle\Phi_{0}^{+}, \mathbf{F} \Psi>\right.}{\left\langle\Phi_{0}^{+}, \mathbf{A} \Psi>\right.} \rho=1-\frac{1}{k} \quad \beta=\frac{<\Phi_{0}^{+}, \mathbf{F}_{d} \Psi>}{\left\langle\Phi_{0}^{+}, \mathbf{F} \Psi>\right.} \ell=\frac{\left\langle\Phi_{0}^{+}, \frac{1}{v} \Psi\right\rangle}{\left\langle\Phi_{0}^{+}, \mathbf{F} \Psi>\right.}
$$

And in the right-hand side of the precursor equations in Eq. (8):

$$
<\Phi_{0}^{+}, \chi_{d}^{(i)} v_{d}^{(i)} \Sigma_{f} \Psi>=\frac{<\Phi_{0}^{+}, \chi_{d}^{(i)} v_{d}^{(i)} \Sigma_{f} \Psi>}{<\Phi_{0}^{+}, \mathbf{F} \Psi>} \frac{<\Phi_{0}^{+}, \mathbf{F} \Psi>}{<\Phi_{0}^{+}, \frac{1}{v} \Psi>} S=\frac{\beta^{(i)}}{\ell} S
$$

$\beta^{(i)}$ is the delayed neutron fraction associated to precursor family number $i$. We define also an effective delayed neutron precursor concentration for family number $i$ by:

$$
\tilde{C}^{(i)}=<\Phi_{0}^{+}, \chi_{d}^{(i)} C^{(i)}>
$$

Finally the PK equations may be written as:

$$
\left\{\begin{array}{l}
\frac{d S}{d t}=\frac{\rho-\beta}{\ell} S+\sum_{i=1}^{D} \lambda^{(i)} \tilde{C}^{(i)} \\
\frac{d \tilde{C}^{(i)}}{d t}=\frac{\beta^{(i)}}{\ell} S-\lambda^{(i)} \tilde{C}^{(i)}
\end{array}\right.
$$

The total number of equations is $(1+D)$. At this stage all the involved coefficients are written using the unknown dynamic flux $\Psi$; they can be approximated by using instead of $\Psi$ the static flux $\Phi$ of the associated critical problem, solution of $\left(\frac{\mathbf{F}}{k_{c}}-\mathbf{A}\right) \Phi=0$; here $k_{c}$ is the multiplication factor of the critical (static) problem associated to the operators $\mathrm{F}$ and $\mathrm{A}$ and may differ slightly from the dynamic multiplication factor $k$ defined in Eq.(77) with the real kinetic flux $\Psi$. Finally, the set of approximate point kinetic parameters is:

$$
\begin{array}{cr}
k \approx \frac{\left\langle\Phi_{0}^{+}, \mathrm{F} \Phi\right\rangle}{\left\langle\Phi_{0}^{+}, \mathrm{A} \Phi>\right.} & \beta \approx \frac{<\Phi_{0}^{+}, \mathrm{F}_{d} \Phi>}{<\Phi_{0}^{+}, \mathrm{F} \Phi>} \\
\beta^{(i)} \approx \frac{\left\langle\Phi_{0}^{+}, \chi_{d}^{(i)} v_{d}^{(i)} \Sigma_{f} \Phi>\right.}{\left\langle\Phi_{0}^{+}, \mathrm{F} \Phi>\right.} & \ell \approx \frac{\left\langle\Phi_{0}^{+}, \frac{1}{v} \Phi>\right.}{\left\langle\Phi_{0}^{+}, \mathrm{F} \Phi>\right.}
\end{array}
$$

\section{References}


Abramowitz, M. and Stegun, I. A., eds., 1964. Handbook of mathematical functions, NBS Applied Mathematics Series 55, National Bureau of Standards, Washington DC $\left(10^{\text {th }}\right.$ printing, 1972)

Aufiero, M., Palmiotti, G., Salvatores, M., Sen, S., 2016. Coupled reactors analysis: New needs and advances using Monte Carlo methodology, Annals of Nuclear Energy 98, 218225

Avery, R. L., 1958. The theory of coupled reactors, Proc. $2^{\text {nd }}$ UN international conference on the peaceful uses of atomic energy, Geneva, Switzerland, vol. 12, p. 182

Bosio, P., Ravetto, P., Rostagno M. M. and Barzilov, A., 2001. Multipoint methods in nuclear reactor kinetics, Proc. Int. Mtg. Mathematical Methods for Nuclear Applications, Salt Lake City, Utah, September 9-13, 2001, American Nuclear Society

Crank, J. and Nicolson, P., 1996. A practical method for numerical evaluation of solutions of partial differential equations of heat-conduction type, Advances in Computational Mathematics 6, 207-226 (reprint of the original 1947 article)

Ganapol, B. D., 2013. A highly accurate algorithm for the solution of the point kinetics equations, Annals of Nuclear Energy 62, 564-571

Henry, A. F., 1958. The application of reactor kinetics to the analysis of experiments, Nuclear Science and Engineering 3, 52-70

Hindmarsh, A. C., 1983. ODEPACK, a systematized collection of ODE solvers, in Scientific Computing, R. S. Stepleman et al., Eds. (North-Holland, Amsterdam, 1983), 55-64

Kobayashi, K., 1992. Rigorous derivation of multi-point kinetic equations with explicit dependence on perturbation, Journal of Nuclear Science and Technology 29(2), 110-120

Komata, M., 1969. On the derivation of Avery's coupled reactor kinetics equations, Nuclear Science and Engineering 38, 193-204

Laureau, A., Buiron, L. and Fontaine, B., 2017a. Local correlated sampling Monte Carlo calculations in the TFM neutronics approach for spatial and point kinetics applications, EPJ Nuclear Science and Technology 3,16

Laureau, A., Buiron, L. and Fontaine, B., 2017b. Towards spatial kinetics in a low void effect sodium fast reactor: core analysis and validation of the TFM neutronic approach, EPJ Nuclear Science and Technology 3,17

Leppänen, J., et al., 2014. Calculation of effective point kinetics parameters in the SERPENT-2 Monte-Carlo code, Annals of Nuclear Energy 65, 272-279

Palmiotti, G., et al., 2018. Investigating decoupling effects in CFV type of reactors using Avery's coupled reactors theory, Proc. Int. Conf. PHYSOR 2018, Cancun, Q.R., Mexico, April 22-26, 2018 
Ravetto, P., et al., 2004. Application of the Multipoint Method to the Kinetics of Accelerator-Driven Systems, Nuclear Science and Engineering 148, 79-88

Ruggieri, J.M., et al., 2006. ERANOS2.1: international code system for GEN-IV fast reactor analysis, Proc. Int. Conf. ICAPP'06, Reno, NV, USA, June 4-8, 2006, paper 6360

Santamarina, A. et al., 2009. The JEFF-3.1.1 Nuclear Data Library - Validation Results from JEF-2.2 to JEFF-3.1.1, JEFF Report 22, NEA No. 6807, Nuclear Energy Agency, OECD.

Scilab Enterprises (2012). Scilab: free and open source software for numerical computation (Version 5.5.2) [Software]. Available from: http://www.scilab.org

Varaine, F., et al. (2012), Pre-conceptual design study of ASTRID core, Proc. Int. Conf. ICAPP'12, Chicago, IL, USA, June 24-28, 2012, paper 12173 